\title{
INTERACTION OF RECOILING SUPERMASSIVE BLACK HOLES WITH STARS IN GALACTIC NUCLEI
}

\author{
Shuo Li ${ }^{1,4}$, F. K. Liu ${ }^{1}$, Peter Berczik ${ }^{3,4,5}$, Xian Chen ${ }^{2}$, and Rainer Spurzem ${ }^{3,4,2}$
}

\begin{abstract}
Supermassive black hole binaries (SMBHBs) are the products of frequent galaxy mergers. The coalescence of the SMBHBs is a distinct source of gravitational wave $(\mathrm{GW})$ radiation. The detections of the strong GW radiation and their possible electromagnetic counterparts are essential. Numerical relativity suggests that the post-merger supermassive black hole ( $\mathrm{SMBH}$ ) gets a kick velocity up to $4000 \mathrm{~km} \mathrm{~s}^{-1}$ due to the anisotropic GW radiations. Here we investigate the dynamical co-evolution and interaction of the recoiling SMBHs and their galactic stellar environments with one million direct $N$-body simulations including the stellar tidal disruption by the recoiling SMBHs. Our results show that the accretion of disrupted stars does not significantly affect the SMBH dynamical evolution. We investigate the stellar tidal disruption rates as a function of the dynamical evolution of oscillating SMBHs in the galactic nuclei. Our simulations show that most of stellar tidal disruptions are contributed by the unbound stars and occur when the oscillating SMBHs pass through the galactic center. The averaged disruption rate is $\sim 10^{-6} \mathrm{M}_{\odot} \mathrm{yr}^{-1}$, which is about an order of magnitude lower than that by a stationary SMBH at similar galactic nuclei. Our results also show that a bound star cluster is around the oscillating SMBH of about $\sim 0.7 \%$ the black hole mass. In addition, we discover a massive cloud of unbound stars following the oscillating SMBH. We also investigate the dependence of the results on the SMBH masses and density slopes of the galactic nuclei.
\end{abstract}

\footnotetext{
${ }^{1}$ Astronomy Department, Peking University, 100871 Beijing, China; fkliu@bac.pku.edu.cn, lis@bac.pku.edu.cn, chenx@bac.pku.edu.cn

${ }^{2}$ Kavli Institute for Astronomy and Astrophysics, Peking University, 100871 Beijing, China

${ }^{3}$ National Astronomical Observatories of China, Chinese Academy of Sciences, 20A Datun Lu, Chaoyang District, 100012, Beijing, China

${ }^{4}$ Astronomisches Rechen-Institut, Zentrum für Astronomie, Universität Heidelberg, Mönchhofstr. 12-14, D-69120 Heidelberg, Germany

${ }^{5}$ Main Astronomical Observatory, National Academy of Sciences of Ukraine, 27 Akademika Zabolotnoho St., 03680 Kyiv, Ukraine
} 
Subject headings: galaxies: active — galaxies: evolution — galaxies: kinematics and dynamics — galaxies: nuclei — methods: numerical

\section{INTRODUCTION}

Supermassive black hole binaries (SMBHBs) are predicted by the hierarchical galaxy formation model in $\Lambda$ cold dark matter $(\Lambda \mathrm{CDM})$ cosmology (Begelman et al. 1980; Volonteri et al. 2003). For merging galaxies, their two SMBHs with galactic cores will firstly approach each other by dynamical friction, and then get close enough to form a bound, compact binary system. After that, the SMBHB may stall at a so called "hard binary separation" for a time even longer than the Hubble time (Begelman et al. 1980). However, recent investigations suggest that the hardening rates of SMBHBs can be boosted and they may coalesce within the Hubble time either due to various stellar dynamical processes other than spherical twobody relaxation (Yu 2002; Chatterjee et al. 2003; Merritt \& Poon 2004; Berczik et al. 2006; Sesana et al. 2008), or gas dynamics (Gould \& Rix 2000; Colpi \& Dotti 2009, and references therein).

Since the evolution of SMBHBs deeply impacts the evolution of host galaxies, it is very important for us to find observational evidences to constrain evolution models of SMBHBs. A statistic way is the calculation of tidal disruption rates. A dormant SMBH could be temporarily activated by tidally disrupting a star passing by and accreting the disrupted stellar debris (Hills 1975; Rees 1988; Evans \& Kochanek 1989; Lodato et al. 2009), which may have been observed in several non-active galaxies Komossa \& Bade 1999; Komossa 2002; Gezari et al. 2008, 2009). Chen et al. (2008) and Chen et al. (2009) calculated the tidal disruption rate in SMBHB systems at different evolutionary stages, and found that is significantly different from the typical rate in a single SMBH for several orders of magnitude.

The most certain proof for detecting SMBHB individually may come from gravitational wave (GW) radiation observation. Coalescing SMBHBs are important sources of GW radiation (Peters 1964; Begelman et al. 1980), and can be detected within the next decades by the Laser Interferometer Space Antenna (LISA) and the Pulsar Timing Array (PTA) program (Berentzen et al. 2009; Amaro-Seoane et al. 2010). Because of the poor spatial resolution of both LISA and PTA for locating GW radiation sources, it is of key importance to detect electromagnetic counterparts (EMCs) of GW radiation sources. Besides, identifying SMBHBs by their EMCs is also essential to constrain the poorly understood galaxy-merger history.

Several EMCs have been suggested in the literature to probe SMBHBs, (1) preces- 
sion of jet orientation and its acceleration in radio galaxies during the in-spiraling of SMBHBs (Begelman et al. 1980; Liu \& Chen 2007), (2) optical periodic outbursts in blazars due to the interaction of SMBHBs and accretion disk (Sillanpaa et al. 1988; Liu et al. 1995, 2006; Liu \& Wu 2002; Valtonen et al. 2008; Haiman et al. 2009), (3) jet reorientation in $\mathrm{X}$-shaped radio galaxies due to the exchange of angular momentum between SMBHBs and accretion disk (Liu 2004), (4) interruption of tidal disruption flares in SMBHBs systems (Liu et al. 2009). Besides, there are also some EMCs to probe the coalescence of SMBHBs and its remnant, (1) intermittent activity in double-double radio galaxies at binary coalescence (Liu et al. 2003), (2) X-ray, UV, optical, and IR afterglow following binary coalescence (Milosavliević \& Phinney 2005; Shields \& Bonning 2008; Lippai et al. 2008; Schnittman \& Krolik 2008), (3) systematically shifted broad emission lines relative to narrow emission lines (Komossa et al. 2008) and off-center active galactic nuclei (AGNs) (Madau \& Quataert 2004; Loeb 2007) because of SMBH GW radiation recoil, (4)tidal disruption flares and hypercompact stellar systems for recoiling black holes (Komossa \& Merritt 2008; O'Leary \& Loeb 2009; Merritt et al. 2009; Stone \& Loeb 2011).

The breakthrough on numerical relativity in the past few years reveals the final stage of SMBHB's coalescence (Pretorius 2005; Campanelli et al. 2006; Baker et al. 2006a). The coalescence remnant SMBH can be recoiled due to the anisotropically GW emission during the inspiral and final coalescence (Peres 1962). For nonspinning SMBHs, the recoil velocity is $V_{\text {kick }} \lesssim 200 \mathrm{~km} \mathrm{~s}^{-1}$ (Baker et al. 2006b; González et al. 2007a; Herrmann et al. 2007), which is just as the same order of magnitude as the stellar dispersion velocity in the galactic center. However, if both of two SMBHs are rapidly spinning, the recoil velocity can be as large as thousands kilometers per second. This recoil velocity depends sensitively on the intersection angle between spin vectors of two SMBHs and their linear momenta. For some extreme cases, with maximally spinning equal-mass SMBHs and antialigned spins in orbital plane, the recoiling velocity can achieve to $\sim 4000 \mathrm{~km} \mathrm{~s}^{-1}$ (Campanelli et al. 2007; González et al. $2007 \mathrm{~b})$.

A recoiling SMBH with high velocity has significant displacement relative to the galactic center. For most of massive galaxies with escape velocity $\lesssim 3000 \mathrm{~km} \mathrm{~s}^{-1}$ (Merritt et al. 2004), the recoiling SMBH has opportunity to escape from its host galaxy. Those recoiling SMBHs which are still bound to host galaxies will oscillate around the galactic centers and change the core density profiles of the host galaxies due to dynamical friction (Boylan-Kolchin et al. 2004; Gualandris \& Merritt 2008). In addition, the recoiling SMBH with a fraction of its accretion disk can produce sets of emission lines separated by relative velocity (Komossa et al. 2008) or off-center active galactic nuclei (AGNs) (Madau \& Quataert 2004; Loeb 2007).

In gas poor environment, a recoiling SMBH might be observed as a "hypercompact stel- 
lar system" (HCSS). That is a kind of star cluster which is bound to the recoiling SMBH, with similar luminosity as a globular cluster or ultracompact dwarf galaxy, and very high velocity dispersion (Merritt et al. 2009; O'Leary \& Loeb 2009). The other signature is an offset tidal flare due to the recoiling SMBH tidally disrupting surrounding stars. Komossa \& Merritt (2008) have used both analysis and $N$-body simulations to calculate the tidal disruption rates of the recoiling SMBH for bound and unbound stars. They found that the tidal disruption rates, in most cases, are smaller than in stationary systems. Recently, O'Leary \& Loeb (2011) have investigated the bound stellar density profile and tidal disruption evolution around the recoiling SMBH. They found that the tidal disruption rate will monotonically fall as a power-law after the recoil. There is also an analytical work focusing on the tidal disruption rate during a short period just after coalescence (Stone \& Loeb 2011), which predicts multiple tidal disruption flares in few years or decades after the coalescence.

Most of the above works about tidal disruption of the recoiling SMBH have focused on theoretical analysis and neglected the impact of background stars. Since the real evolution process in such kind of system is very complex, a detailed study on the recoiling $\mathrm{SMBH}$ co-evolving with the galactic center is essential. Unlike previous work, we evolve the entire system before and after the recoil with unbound stars included. Our work focuses on investigating the co-evolution between the recoiling SMBH and surrounding stars with tidal disruption and accretion processes. To it come true, we use a special high-accuracy, parallel direct $N$-body code accelerated by many-core hardware ( $\varphi$-GRAPE and $\varphi$-GPU) (Berczik et al. 2005; Harfst et al. 2007; Spurzem et al. 2009; Just et al. 2011), including a simplified tidal disruption scheme (Fiestas et al. 2011). Most of our simulations are calculated on the laohu GPU cluster in National Astronomical Observatories of China (NAOC). With up to million of particles in simulation, we can find out the dynamical evolution of stars near the recoiling SMBHs and estimate their tidal disruption rates, which may give us some hints for observation.

Our results show that the accretion of disrupted stars does not significantly impact the dynamical evolution of recoiling SMBH. However, it changes the tidal disruption rate compared to a stationary SMBH system in a galactic center. Most of tidal disruption events are contributed by unbound stars when the SMBH passing through the galactic center, and the disruption rate in average is $\sim 10^{-6} \mathrm{M}_{\odot} \mathrm{yr}^{-1}$, which is roughly an order of magnitude lower than the stationary case, $\sim 10^{-5} \mathrm{M}_{\odot} \mathrm{yr}^{-1}$. Besides, the tidal disruption rate for oscillating $\mathrm{SMBH}$ far from the galactic center is roughly one or two orders of magnitude lower than stationary SMBH. We also find a bound stellar system around the recoiling SMBH with a mass of $\sim 0.7 \%$ black hole mass, which is consistent with Merritt et al. (2009). Except for bound stellar systems, we find that there is a "polarization cloud" composed of stars dynamically perturbed by the oscillating SMBH. This "polarization cloud" is axisymmetri- 
cally diffuse in a large spatial area which is beyond the gravitational influence region of the oscillating SMBH. For this reason, most of the stars in the cloud are not permanently bound to the SMBH. This kind of clouds are the echo of dynamical friction. The calculations for parameter dependence show that the variation of initial mass of the recoiling SMBH can speed up or slow down the dynamical evolution, and impact the tidal disruption rate. In addition, the variation of density slope can obviously change the dynamical evolution and tidal disruption rate of the recoiling SMBH.

We give our galactic model and simulation method in Section 2. Some results about bound/unbound stellar systems and tidal disruption rates for the stationary/recoiling SMBH are showed in Section 3. The dependence of our results on other parameters are also investigated. Section 4 gives discussion about the observational implications and a short summary.

\section{GALACTIC MODEL AND $N$-BODY SIMULATIONS}

\subsection{Galactic Model}

For simplicity, we adopt a spherical Dehnen model to describe the ellipticals or bulges of galaxies (Dehnen 1993), which is different from a Sérsic model used in Gualandris \& Merritt (2008). In a Dehnen model, the space density profile follows

$$
\rho(r)=\frac{3-\gamma}{4 \pi} \frac{M a}{r^{\gamma}(r+a)^{4-\gamma}},
$$

where $a$ is the scaling radius, $M$ is the total mass of galaxies, and the slope index $\gamma$ is a constant between interval [0,3). The mass distribution is proportional to $r^{-\gamma}$ for $r \ll a$ and

$r^{-4}$ for $r \gg a$. In this model, it is easy for us to derive the galactic potential, cumulative mass, and half-mass radius respectively (Dehnen 1993),

$$
\begin{gathered}
\Phi(r)=\frac{G M}{a} \times\left\{\begin{array}{lc}
-\frac{1}{2-\gamma}\left[1-\left(\frac{r}{r+a}\right)^{2-\gamma}\right] & \text { for } \gamma \neq 2 \\
\ln \frac{r}{r+a} & \text { for } \gamma=2
\end{array}\right. \\
M(r)=M\left(\frac{r}{r+a}\right)^{3-\gamma}, \\
r_{1 / 2}=a\left[2^{1 /(3-\gamma)}-1\right]^{-1} .
\end{gathered}
$$


where $G$ is the gravitational constant. From Equation (2) the escape velocity at radius $r$ for $\gamma<2$ is

$$
\begin{aligned}
V_{\mathrm{esc}}(r) & =\sqrt{|2 \Phi(r)|} \\
& =\left(\frac{2}{2-\gamma} \frac{G M}{a}\right)^{1 / 2}\left|1-\left(\frac{r}{r+a}\right)^{2-\gamma}\right|^{1 / 2}, \text { for } \gamma<2 .
\end{aligned}
$$

Thus the escape velocity from the galactic center for $\gamma<2$ is

$$
V_{\mathrm{esc}}(0)=\left(\frac{2}{2-\gamma} \frac{G M}{a}\right)^{1 / 2} .
$$

If there is a $\mathrm{SMBH}$ with mass $M_{\mathrm{BH}}$ at the galactic center, we can estimate its influence radius $r_{\text {inf }}$ by using the definition with stellar mass $M_{\star}\left(r \leqslant r_{\text {inf }}\right)=2 M_{\mathrm{BH}}$ as in Merritt (2006) 1

$$
r_{\mathrm{inf}}=\frac{a\left(2 M_{\mathrm{BH}} / M\right)^{\frac{1}{3-\gamma}}}{1-\left(2 M_{\mathrm{BH}} / M\right)^{\frac{1}{3-\gamma}}} .
$$

For simplicity, we adopt model units $G=M=a=1$ thereafter. In this new unit, the quantities relate to physical quantities with the scale factor of time, velocity, and length, respectively

$$
\begin{aligned}
{[T] } & =\left(\frac{G M}{a^{3}}\right)^{-1 / 2} \\
& =1.491 \times 10^{6}\left(2^{\frac{1}{3-\gamma}}-1\right)^{3 / 2}\left(\frac{M}{10^{11} \mathrm{M}_{\odot}}\right)^{-1 / 2}\left(\frac{r_{1 / 2}}{1 \mathrm{kpc}}\right)^{3 / 2} \mathrm{yr}, \\
{[V] } & =\left(\frac{G M}{a}\right)^{1 / 2} \\
& =655.8 \times\left(2^{\frac{1}{3-\gamma}}-1\right)^{-1 / 2}\left(\frac{M}{10^{11} \mathrm{M}_{\odot}}\right)^{1 / 2}\left(\frac{r_{1 / 2}}{1 \mathrm{kpc}}\right)^{-1 / 2} \mathrm{~km} \mathrm{~s}^{-1}, \\
{[R] } & =a=\left(2^{\frac{1}{3-\gamma}}-1\right)\left(\frac{r_{1 / 2}}{1 \mathrm{kpc}}\right) \mathrm{kpc} .
\end{aligned}
$$

\footnotetext{
${ }^{1}$ This kind of definition is validated only for a singular isothermal sphere nucleus. Here we adopt it also for $\gamma \neq 2$ to estimate $r_{\text {inf }}$ at an order of magnitude.
} 
With the model units, Equations (44), (6), and (7) can be rewritten

$$
\begin{gathered}
r_{1 / 2}=\left[2^{1 /(3-\gamma)}-1\right]^{-1}, \\
V_{\mathrm{esc}}(0)=\left(\frac{2}{2-\gamma}\right)^{1 / 2}, \\
r_{\mathrm{inf}}=\frac{\left(2 M_{\mathrm{BH}}\right)^{\frac{1}{3-\gamma}}}{1-\left(2 M_{\mathrm{BH}}\right)^{\frac{1}{3-\gamma}}} \approx\left(2 M_{\mathrm{BH}}\right)^{\frac{1}{3-\gamma}} .
\end{gathered}
$$

For a fiducial model adopted in the following calculations with $\gamma=0.5, M=4 \times 10^{10} \mathrm{M}_{\odot}$, $M_{\mathrm{BH}}=4 \times 10^{7} \mathrm{M}_{\odot}$ and $r_{1 / 2}=1 \mathrm{kpc}$, we can derive $V_{\mathrm{esc}}(0) \simeq 847 \mathrm{~km} \mathrm{~s}^{-1}$ and $r_{\mathrm{inf}} \simeq 29 \mathrm{pc}$.

\subsection{Tidal Disruption Scheme in $N$-Body Simulation}

Compared to a scattering experiment, direct $N$-body simulation can well represent the dynamical co-evolution of the recoiling SMBH and surrounding stars, especially for the stellar interactions. However, it can not deal with other process well, for example, the tidal disruption by SMBH. To solve this problem, we implement a special tidal disruption scheme in our simulation.

A star with mass $m_{*}$ and radius $r_{*}$ will be tidally disrupted if it approaches a BH within the tidal radius (Hills 1975; Rees 1988)

$$
r_{\mathrm{t}} \simeq \mu r_{*}\left(M_{\mathrm{BH}} / m_{*}\right)^{1 / 3} .
$$

Here $\mu$ is a dimensionless parameter of order unity. It is roughly $10^{-6} r_{\text {inf }}-10^{-5} r_{\text {inf }}$ for the real conditions in galactic nuclei (Merritt 2006). Based on Equation (14), to make sure that the tidal radius is larger than the event horizon of the $\mathrm{BH}$, there is a limitation for $M_{\mathrm{BH}}$. For a disrupted star with solar radius $\mathrm{R}_{\odot}$ and mass $\mathrm{M}_{\odot}$, a Schwarzschild $\mathrm{BH}$ should have $M_{\mathrm{BH}} \lesssim 10^{8} \mathrm{M}_{\odot}$. This limitation mass can be heavier when the $\mathrm{BH}$ has rapid spin.

In order to investigate the tidal disruption of the recoiling SMBH with $N$-body simulation, we have to find a proper way to represent the tidal radius in our simulations. As shown in Equation (14), the tidal radius is proportional to the radius of the disrupted star. However, in our $N$-body simulations without stellar evolution scheme, stars are point like particles, whose radii are not defined. We can use the approximate scaling relation between 
$r_{\text {inf }}$ and $r_{\mathrm{t}}$ discussed above to set a proper value for $r_{\mathrm{t}}$, but this value is so small that there will be only few tidal disruption events recorded in the simulation. That is because of the limitation on particle resolution. Our simulation particle number is not enough even with $N=10^{6}$.

To solve this problem, a simplified strategy is adopted. We assume a larger $r_{\mathrm{t}}$ in our $N$ body simulation to represent the tidal radius. The particle which get close to $\mathrm{SMBH}$ within $r_{\mathrm{t}}$ will be tidally disrupted and removed from the system with its mass added onto the SMBH. With this method, we can directly follow the growth of $M_{\mathrm{BH}}$ and calculate the tidal disruption rate. However, the large $r_{\mathrm{t}}$ we adopted here will overestimate the tidal disruption rate. The solution is to carry out a series of simulations with decreasing $r_{\mathrm{t}}$ and extrapolate the results to the regime corresponding to real galaxies. Our extrapolation scheme is explained in Section 3.3.1 in detail. We do not consider the growth of $r_{\mathrm{t}}$ for accreting SMBH or the momentum transfer from tidal disrupted stars to the recoiling SMBH, because the total mass of disrupted stars is negligible compared to the BH. A test simulation taking into account momentum transfer shows no significant difference in either the dynamical evolution of recoiling SMBH or the stellar disruption rate.

\subsection{Numerical Method}

To investigate the evolution of the recoiling $\mathrm{SMBH}$ with tidal disruption carefully, we make a series of integrations with different parameters which are listed in Table 1. The first column is the sequence number for different models. Columns (2) - (4) give particle number $N$, tidal radius $r_{\mathrm{t}}$, and initial stellar density slope $\gamma$ respectively. Column (5) is the initial mass ratio between $M_{\mathrm{BH}}$ and the total mass of system. Column (6) gives initial recoil velocity in the unit of escape velocity at the galactic center.

All of our integrations adopt a parallel direct $N$-body $\varphi$-GRAPE/ $\varphi$-GPU code with fourth-order Hermite integrator and simplified tidal disruption scheme. They all have timestep accuracy parameter $\eta=0.01$ and a softening length $\epsilon=10^{-5}$. Most of our models adopt $N=10^{6}$ equal mass particles, with initial mass of SMBH $M_{\mathrm{BH}}=0.001$ to represent the ellipticals/bulges and central SMBH respectively. To find out the dependency of the results on particle number, several sets of integrations with $50 \mathrm{~K}$ and $250 \mathrm{~K}$ particles have been included. There is also a calculation with $M_{\mathrm{BH}}=0.002$ to check the dependency on $M_{\mathrm{BH}}$. As mentioned before, for the purposes of extrapolating to the smaller tidal radius in the real galactic center conditions, we have varied the $r_{\mathrm{t}}$ from $5 \times 10^{-5}$ to $5 \times 10^{-2}$, which is roughly corresponding to $\sim 10^{-3} r_{\text {inf }}$ to $\sim r_{\text {inf }}$ for $\gamma=0.5$. Since the density slope prior to the recoil is under debate, we choose $\gamma=0.5$ as our fiducial value, and also investigate 
other cases with $\gamma=1.0$ and 1.5. The recoil velocity $V_{\mathrm{k}}$ is set to $V_{\mathrm{k}}=0.7 V_{\text {esc }}$ for most of the integrations, and there are calculations without recoiling velocity for comparison. Besides, an integration with $V_{\mathrm{k}}=1.1 V_{\mathrm{esc}}$ and $\gamma=1.0$ is included for comparing with Komossa \& Merritt (2008).

Since Dehnen model provides the stellar distribution in a galaxy without central SMBH, we have to first to set up consistent initial conditions for our system. At first, we put a SMBH into the center of a stellar system with Dehnen profile, and evolve the entire system for 50 simulation time units to relax the core region. After that the system is dynamically and thermally relaxed in relation to the $\mathrm{SMBH}$ gravitational potential. At this stage, we do not consider stellar tidal disruption process. However, we want to make sure that stars inside the tidal disruption loss cone of the central SMBH have already been removed by tidal accretion before the recoil of SMBH. Therefore, as a second step, we switch on the tidal disruption scheme in the program and run it again with the SMBH still in equilibrium in the center this costs a few dynamical timescales at the influence radius. Thus all particles within the tidal disruption loss cone can be removed before we apply a kick to the SMBH. After setting up the initial configuration of the stellar distribution of the systems, we artificially give a recoil velocity to the $\mathrm{SMBH}$ and follow its dynamical evolution.

\section{RESULTS}

\subsection{Dynamical Evolution of the recoiling SMBH}

To carefully investigate the dynamical evolution of recoiling SMBH, we have a long time integration with model 06 to $t=200$, as shown in Figure 1. It is clear that the evolution of the recoiling SMBH can be easily divided into two different phases. The phase I has obviously damping trajectory from $t=0$ to $t \sim 50$. After that, the SMBH evolves into phase II, where the trajectory damped very slow for a long time. In phase I, the trajectory of recoiling

SMBH can be well predicted by Chandrasekhar's dynamical friction theory (Chandrasekhar 1943). However, this stage will just keep till the SMBH's oscillation decayed to the size of core, where a slowly decayed phase II begins. That is because the stellar mass interior to SMBH's orbit is roughly equal to $M_{\mathrm{BH}}$ at the end of phase I, where the assumptions of Chandrasekhar's dynamical friction theory are invalid. We have tried to fit the trajectory analytically in phase I with the same method as Gualandris \& Merritt (2008) used, and got a similar result even though a Dehnen model is adopted here instead of their core-Sérsic model.

If we continue the integration, the orbital oscillation of the SMBH will slowly decay and 
finally achieve to a thermal equilibrium with surrounding stars, which is the so-called phase III in Gualandris \& Merritt (2008). Here we have not continue our integrations into that stage because phase III is actually similar to the case of a stationary central SMBH, which is out of the scope of this paper. We will just focus on phase I and phase II in the following integrations. Thus all of our models integrate to $t=100$ in order to make sure that the evolution can achieve to phase II.

With regard to the orbital motion of the $\mathrm{SMBH}$, our results agree qualitatively well with those of Gualandris \& Merritt (2008). That is because the growth of the black hole due to tidal accretion, which did not take into account in Gualandris \& Merritt (2008), is relatively small.

\subsection{Compact Star Clusters around the Recoiling SMBHs}

A recoiling SMBH can carry a retinue of bound stars. Merritt et al. (2009) predict that there will be a "hypercompact stellar system" (HCSS) bound to the recoiling SMBH. We can analytically estimate the bound population of the HCSS with Equations (1a) and (6a) in Merritt et al. (2009). For example, the roughly boundary radius $r_{\mathrm{ej}}$ of HCSS should be

$$
r_{\mathrm{ej}} \equiv \frac{G M_{\mathrm{BH}}}{V_{\mathrm{ej}}^{2}},
$$

with our Model 06, where $V_{\mathrm{ej}}=0.7 V_{\mathrm{esc}} \approx 0.8$, we have $r_{\mathrm{ej}} \approx 1.5625 \times 10^{-3}$ in our simulation unit. Thus we can define a factor $f_{\mathrm{b}}$ to describe the fraction of bound stars,

$$
f_{\mathrm{b}} \equiv \frac{M_{\mathrm{b}}}{M_{\mathrm{BH}}}=F_{1}(\gamma)\left(\frac{r_{\mathrm{ej}}}{r_{\mathrm{inf}}}\right)^{3-\gamma},
$$

where $M_{\mathrm{b}}$ is total mass of bound stars. Based on Equations (15) and (13), we have $f_{\mathrm{b}} \approx$ $3.8845 \times 10^{-5} F_{1}(\gamma)$. For the case of $\gamma=0.5$, with $F_{1}(0.5)$ in Figure 1a of Merritt et al. (2009), we have $f_{\mathrm{b}} \sim 0.001$. That means the ratio for HCSS mass to $M_{\mathrm{BH}}$ should be $\sim 0.1 \%$, corresponding to just few particles for $1 \mathrm{M}$ simulation. It should be aware that this fraction is just a lower limit because our "two times $M_{\mathrm{BH}}$ " assumption actually overestimate $r_{\mathrm{inf}}$. To confirm the conclusion above, we investigate this problem with $N$-body simulations.

In the simulation, it is very difficult to distinguish whether a star is bound to SMBH or not. That is because most of star particles with negative total energies relative to the $\mathrm{SMBH}$ are loosely bound. Only few of them could orbit the SMBH for more than one orbital period. For this reason, we identify a star to be bound to the recoiling SMBH if following two criteria 
are both satisfied: the particle (1) has negative total energy relative to the SMBH and (2) remains bound for at least one orbital oscillation period of the recoiling SMBH. We find that most of the "bound" particles around SMBH particle will be evaporated before the end of simulation. This effect is more significant for our escaping SMBH calculation with model 10, where the number of bound particles decreases monotonically. For other oscillating SMBH calculations, there are many unbound particles can be captured by the SMBH. That means the interaction between the HCSS and stellar background is very important.

Figure 2 shows the evolution of bound stars around a recoiling SMBH in model 06. At $t=0$, when $V_{\mathrm{ej}}=0.7 V_{\mathrm{esc}}$, there are five bound particles, roughly consistent with the estimation from Equation (16). The number of bound particles, $N_{\text {bd }}$, slightly increases during the evolution from phase I to phase II. Because in phase II (1) the low relative velocity between stars and the recoiling SMBH increases the probability of dynamical capture and (2) the stellar density around the SMBH in phase II is higher. Our results suggest that a HCSS may grow via capturing unbound single stars. However, the low particle resolution and large statistical error prevent us from further investigation of the growth of HCSS.

The HCSS may be detected as an off-nuclear compact system of similar size and stellar mass of a globular clusters but of having very high internal velocities (Merritt et al. 2009; O'Leary \& Loeb 2011). However, that is not the whole story. As shown in the Figures 3 and 4, we find that there is a stellar cloud composed of stars which are strongly impacted by the recoiling SMBH at the first apocenter. The total mass of this cloud is comparable to $M_{\mathrm{BH}}$. They form a quasi-axisymmetric stellar cloud and always have a dense region around SMBH. This kind of clouds gives an evidence for the echo of dynamical friction. For this reason, we name that stellar cloud as "polarization cloud". In our simulation of model 06, we choose a time snapshot when the SMBH arrives at its first apocenter, and pick up those polarization cloud members from the $E_{\text {tot }}-r$ phase space which includes all of star particles. All of polarization cloud members in the $E_{\text {tot }}-r$ phase space are outliers around $\mathrm{SMBH}$ particle and can be easily distinguished. Thus we can collect those polarization cloud particles and trace their evolution. However, it is very difficult to find an automatical solution to efficiently distinguish those polarization cloud members for every time snapshot. To deeply investigate this kind of polarization clouds, an efficient method to extract those outlier particles is needed. This problem should be solved in our following work.

In Figure 3, we plot that polarization cloud at time $t=1.863 \mathrm{Myr}$, when the recoiling SMBH arrives at its first apocenter. All the parameters here are obtained from model 06. In order to investigate the origin and evolution of those cloud members, we look back the data and follow the evolution of the stars from time $t=0$. Figure 4 gives the evolution of those stars and the recoiling $\mathrm{SMBH}$ from $t=0$ until $t=42.6 \mathrm{Myr}$, when our computation 
stopped. The snapshots with $t=0 \mathrm{Myr}, 0.852 \mathrm{Myr}$, 1.863 Myr in Figure 4 show the evolutions of the cloud members from beginning toward the first apocenter of the recoiling SMBH. The following three snapshots correspond to the time when the recoiling SMBH comes back from the first apocenter and passes through the density center for the second time. The snapshot at $t=7.984 \mathrm{Myr}$ shows the SMBH passing through galactic center for the third time, and the last snapshot is for the end of computation. Our calculation results and Figure 4 imply that the shape of polarization cloud varies with the oscillations of the recoiling SMBH. The comparison between the first and the last two panels vividly shows the changes of the cloud members during the oscillation.

As shown in Figure 2, there are only small fraction of stars really bound to the SMBH, whereas the mass of the polarization cloud is roughly equal to $M_{\mathrm{BH}}$. Therefore most of these cloud members are unbound to the recoiling SMBH. After the recoil of SMBH, those polarization cloud members are accelerated and fall behind the oscillating SMBH. During the oscillation, some of the stars gradually expand to larger area while others continuously follow the SMBH to oscillate around the galactic center, and form an axisymmetrical distribution along the velocity direction of the recoiling SMBH. After multiple interactions with oscillating $\mathrm{SMBH}$, these stars obtain energy from oscillating SMBH and diffuse to a large area. This is a vivid example that an oscillating $\mathrm{SMBH}$ transfer its orbital energy to surround stars through dynamical friction.

In order to investigate the observational properties of this polarization cloud, we focus on its most compact stage, when the recoiling SMBH arrive at the apocenter for the first time. The distribution of those stars are shown in Figure 3. Here we scale the $N$-body system to our physical fiducial galaxy model with stellar mass $M=4 \times 10^{10} \mathrm{M}_{\odot}$ and half mass radius $r_{1 / 2}=1 \mathrm{kpc}$. For such system, the polarization cloud has mass of $M_{\mathrm{csc}} \simeq 10^{7} \mathrm{M}_{\odot}$, and a size of diameter $D \simeq 260 \mathrm{pc}$. Further calculation shows that, its half mass radius is $\sim 83$ pc. This size and mass are much larger than a globular cluster and comparable to an ultracompact dwarf galaxy. We also calculate the velocity dispersion of this polarization cloud. The line-of-sight velocity dispersion along X, Y and Z axes are $185 \mathrm{~km} \mathrm{~s}^{-1}, 190 \mathrm{~km} \mathrm{~s}^{-1}$ and $189 \mathrm{~km} \mathrm{~s}^{-1}$ individually. That is significantly higher than a typical ultracompact dwarf galaxy. Comparing with bound HCSS, this polarization cloud has larger size, heavier mass and similar velocity dispersion. Since this cloud is mixed with HCSS, it may bring troubles to the detection of HCSS. We also estimate the average surface density inside the half mass radius of the polarization cloud. The overdensity of the polarization cloud relative to the stellar background is only $\sim 4 \%$. This may be because of that the apocenter of the recoiling $\mathrm{SMBH}$ here is not very far from the galactic center, where the surface density of the stellar background is still relatively high in model 06 with $\gamma=0.5$. For a SMBH with a higher recoil velocity and a larger apocenter, the situation may be improved. To investigate this 
problem, more simulations are needed. We will investigate this problem in our future work.

It should be noted that our example above is just for one time snapshot of the evolving SMBH. Every snapshot could have a similar stellar cloud. The composition of these clouds may form an interesting structure which can trace the trajectory of the SMBH. The evolution and observation characters of this kind of structures will be discussed in our future work. For a recoiling SMBH with highly enough velocity to escape from the galaxy, that kind of polarization cloud may not be distinguishable because many stars will be dropped behind the escaping SMBH. Without the multiple interactions between oscillating SMBH and surrounding stars, the polarization cloud can not survive for a long time.

\subsection{Tidal Disruption}

\subsubsection{Calculate Tidal Disruption Rate Numerically}

For a solar type star disrupted by a SMBH with mass $M_{\mathrm{BH}}=4 \times 10^{7} \mathrm{M}_{\odot}$, based on Equation (14) and (10), the tidal radius is $\sim 8 \times 10^{-6} \mathrm{pc}$, corresponding to $\sim 10^{-8}$ in simulation units with our fiducial model. As mentioned in Section 2.1, because of the limitation on particle resolution, it is practically impossible with present computing capabilities to calculate the tidal disruption rate directly (with a realistically small tidal radius) through direct $N$-body simulations.

To solve this problem, we perform a parameterized study, which treats both the particle number $N$ and the size of the tidal disruption radius $r_{\mathrm{t}}$ as free parameters. All of the simulations have been done with these varying parameters, and physical conclusions are drawn from extrapolating to the real galactic conditions. Fiestas \& Spurzem (2010) have shown in Fokker-Planck models that such scaling is reliable and provides physically correct results. Besides, tidal disruption events in our simulations, as well as the case in real galactic center, do not occur frequently, which means that the increase of $M_{\mathrm{BH}}$ is discrete. Thus it is difficult to compute the tidal disruption rate through calculating accreted masses in time bins. Instead, we measure the tidal disruption rate in a cumulative, averaged way - fitting the mass growth of the black hole and derive its time derivative.

In order to find out the extrapolation relation, the dependence of tidal disruption rate on particle numbers and tidal radius should be investigated. Figure 5 shows the disruption rates changes with different $N$ and $r_{\mathrm{t}}$, with parameters are $N=250 \mathrm{~K}, 1 \mathrm{M}, r_{\mathrm{t}}=5 \times 10^{-3}, 1 \times$ $10^{-3}, 5 \times 10^{-4}, 5 \times 10^{-5}, \gamma=0.5, M_{\mathrm{BH}}=0.001$ and $V_{\mathrm{ej}}=0.7 V_{\mathrm{esc}}$. The left panel shows that the dependence of averaged tidal disruption rates on $N$ and $r_{\mathrm{t}}$. Here we calculate the averaged disruption rates for phase I, phase II and entire process separately with different 
$N$ and $r_{\mathrm{t}}$. These integrations show that the dependence of tidal disruption rate on particle number is very weak. Further discussions are showed in Section 3.4.

Based on the loss cone feeding theory (Frank \& Rees 1976; Lightman \& Shapiro 1977), contrary to empty loss cone case, the tidal disruption rate for full loss cone does not depend on $N$. It should be notice that the tidal disruption rate here is for mass disruption rate, and the disrupted particles indeed depend on $N$. The slight different average rates between $1 \mathrm{M}$ and $250 \mathrm{~K}$ cases means that the loss cones in our integrations are nearly full and the changes of particle numbers do not bring significant impact.

Opposed to particle numbers, the changes on $r_{\mathrm{t}}$ strongly influence the disruption rates. A detailed study is in the right panel of Figure 5, which shows the evolution of disruption rate $-r_{\mathrm{t}}$ dependence for several special points with integrations for $N=1 \mathrm{M}$. Five special snapshot have been picked out. Here "1st. APO.", "1st. BACK", "1st. DOWN", "2nd APO." and "Phase Tran." refer respectively to the snapshot that the oscillating SMBH the first time arrives at the apocenter, the first time returns to the density center, the first time reaches the opposite apocenter, the second time return to the apocenter and the time of transition from phase I to phase II. Our results show that the disruption rates increase for every $r_{\mathrm{t}}$ value during the evolution of the recoiling SMBH. That is because a decayed orbit of SMBH leads to a growing stellar background around it. Besides, the similar linear disruption rate $-r_{\mathrm{t}}$ relation as left panel has been found. However, the relation here for the first four snapshots are not as good as the average results in the left panel. That may because our particle resolution for small $r_{\mathrm{t}}$ is not good enough in phase I.

Based on our result data, it seems that there is an approximate linear relation between $r_{\mathrm{t}}$ and disruption rates. As a result of full loss cone condition, the disruption rates will be proportional to cross section $S$ and density $\rho(r)$ near the recoiling SMBH,

$$
\dot{M} \simeq S \rho(r) \bar{v}_{\text {rela }},
$$

where $\bar{v}_{\text {rela }}$ is average relative velocity between the recoiling SMBH and the surrounding stars.

It is difficult for us to analytically derive stellar distribution around a recoiling SMBH. However, the cross section can be wrote as

$$
S \simeq \pi r_{\mathrm{t}}^{2}\left(1+\frac{2 G M_{\mathrm{BH}}}{r_{\mathrm{t}} \bar{v}_{\text {rela }}^{2}}\right),
$$

which can write the tidal disruption rate as 


$$
\dot{M} \simeq \pi r_{\mathrm{t}}^{2} \rho(r) \bar{v}_{\text {rela }}\left(1+\frac{2 G M_{\mathrm{BH}}}{r_{\mathrm{t}} \bar{v}_{\text {rela }}^{2}}\right) .
$$

For $r \gg r_{\mathrm{t}}$ that is $2 G M_{\mathrm{BH}} / r_{\mathrm{t}} \gg \bar{v}_{\text {rela }}^{2}$ (even if one takes into account that $\bar{v}_{\text {rela }}^{2}$ contains a contribution from the SMBH kick velocity), so we are in the gravitational focusing regime, where

$$
S \simeq \frac{2 \pi G M_{\mathrm{BH}}}{\bar{v}_{\text {rela }}^{2}} r_{\mathrm{t}} .
$$

Thus we have

$$
\dot{M} \simeq \frac{2 \pi G M_{\mathrm{BH}}}{\bar{v}_{\text {rela }}} \rho(r) r_{\mathrm{t}} .
$$

If $\rho$ and $\bar{v}_{\text {rela }}$ are constant with time, the tidal accretion rate will scale linearly with $r_{\mathrm{t}}$. This relation is clearly only correct in a time averaged sense, because when the SMBH oscillating in the galaxy, all quantities will be strongly changed within a single orbit (density, stellar velocity dispersion and SMBH velocity, the two latter quantities determining $\bar{v}_{\text {rela }}$ ). However, in a running time averaged over several orbits, our results show that there is not a large variation in these quantities. So we can define a dimensionless tidal accretion parameter $\alpha$ by the following relation:

$$
t_{\mathrm{dyn}} \frac{\dot{M}}{M_{\mathrm{BH}}}=\alpha \frac{r_{\mathrm{t}}}{\bar{v}_{\text {rela }} t_{\mathrm{dyn}}} .
$$

Here $t_{\mathrm{dyn}}$ is the dynamic time scale around the position of the recoiling SMBH. Our simulations imply that across all of our model families $\alpha$ does not depend strongly on $N$ or $r_{\mathrm{t}}$, but only on the evolutionary phase. Thus $\alpha$ can be used in extrapolations to the real system with very small $r_{\mathrm{t}}$ and large $N$. Based on the analysis above, though it is complicated to get an accurate scaling relation, we still can estimate the tidal disruption rate through linear extrapolation of tidal radius.

\subsubsection{Tidal Disruption Rate for the Stationary SMBH}

For comparison purposes, we calculate the tidal disruption rate of a stationary SMBH without recoil in the galactic center. A series of simulations with different $r_{\mathrm{t}}$ values are carried out. Figure 6 shows the static disruption rate for $r_{\mathrm{t}}=5 \times 10^{-3}, 1 \times 10^{-3}, 5 \times 10^{-4}, 5 \times 10^{-5}$, corresponding to model 15, 17, 05 and 19 respectively. As mentioned in Section 3.3.1, the 
tidal radius for our fiducial model is $\sim 8 \times 10^{-6} \mathrm{pc}$, corresponding to $\sim 10^{-8}$ in our simulation units. Our simulation results with different particle numbers for stationary case show that the tidal disruption rates weakly depend on the particle numbers. Thus we can loosely accept a full loss cone condition for the stationary case. As discussed in Section 3.3.1, there is a roughly linear correlation between tidal disruption rate and $r_{\mathrm{t}}$ for full loss cone case. Based on Equation (21), we can extrapolate our simulation results to the real galaxy conditions with smaller tidal radii. Figure [6 shows that the tidal disruption rate for $r_{\mathrm{t}}=10^{-3}$ is $\sim 8 \times 10^{-6}$ in our simulation units. That can be extrapolated to $r_{\mathrm{t}} \sim 10^{-8}$, and gives us the tidal disruption rate $\sim 10^{-10}$, which corresponds to the order of magnitude $\sim 10^{-5} \mathrm{M}_{\odot} \mathrm{yr}^{-1}$ for our fiducial model.

\subsubsection{Tidal Disruption Rate for the recoiling SMBH}

In a recoiling SMBH system, there are two populations of stars contributed to the tidal disruption rate. One is the bound stars around the $\mathrm{SMBH}$, and the other is those unbound stars encountered with the recoiling SMBH. If the bound stars dominate, as demonstrated in Komossa \& Merritt (2008) for an escaping SMBH, the tidal disruption rate should roughly keep a constant level at the beginning, and finally drop down because of the consumption of bound stars. On the contrary, if the tidal disruption rate is dominated by unbound stars, the results will depend on stellar density, relative velocity of SMBH, and the size of tidal radius, which described by Equation (19).

If we just consider about the contribution of the unbound stars, for an oscillating SMBH, its tidal disruption rate near the galactic center should be higher than the apocenter region because of the different stellar density. However, $\bar{v}_{\text {rela }}$ can also change the result. For instance, we have an estimation in our Model 16 with $r_{\mathrm{t}}=5 \times 10^{-3}, \gamma=0.5, M_{\mathrm{BH}}=0.001$ and $V_{\mathrm{ej}}=$ $0.7 V_{\mathrm{esc}} \simeq 0.8$. Our simulation result shows that the first apocenter of the recoiling $\mathrm{SMBH}$ is around $r_{\mathrm{BH}} \sim 1.4$. With the assumptions that $\bar{v}_{\text {rela }}(r=1.4) \sim 0.1$ and $\bar{v}_{\text {rela }}(r=0) \sim 0.8$, we can estimate the disruption rate ratio from the galactic center to the first apocenter region. By substituted into Equations (11) and (19), that ratio is $\sim 8$. When scaling to the real galactic center conditions with $r_{\mathrm{t}} \sim 10^{-8}$, it should be $\sim 3$. Moreover, this ratio also depends on $\bar{v}_{\text {rela }}$. If $\bar{v}_{\text {rela }}$ near apocenters can be larger than 0.1 , that ratio may be higher. Unfortunately, we can not obtain an accurate value for $\bar{v}_{\text {rela }}$. For estimation, it can be seen that the tidal disruption rates near the galactic center are several times or even an order of magnitude higher than the apocenter region.

That conclusion is confirmed by our simulation results. Figure 7 gives the distribution of the tidal disruption events count $N_{\mathrm{td}}$ relative to distance $r$ in model 16. It shows that most 
of the tidal disruption events appear around the density center, which indicates a boosted tidal disruption rate when the oscillating SMBH pass through the galactic center. A SMBH around the apocenter with a nearly zero velocity always corresponds to a low $\bar{v}_{\text {rela }}$, which should give us a high disruption rate. However, as Equation (19) shown, the low stellar density environment makes the tidal disruption events less than at galactic center. The higher disruption rate near the galactic center means that, for an oscillating SMBH, most of tidal disruption events are contributed by unbound stars. For this reason, unbound stars are very important for calculating tidal disruption rate.

Figure 8 shows the tidal disruption rates for a recoiling $\mathrm{SMBH}$ with different $r_{\mathrm{t}}=$ $5 \times 10^{-3}, 1 \times 10^{-3}, 5 \times 10^{-4}, 5 \times 10^{-5}$, which belong to model 16, 18, 06 and 20 respectively. To increase the accuracy of our fitting results for disruption rate in phase I and phase II, we neglect the transition boundary between the two phases in the fitting. Therefore, we fit disruption rate for phase I and phase II separately. As a result, the fitted results are not continuous between two phases.

The left part of Figure 8 shows that the tidal disruption rates in phase I are linearly increasing. For different $r_{\mathrm{t}}$ values, there is also a similar linear relation to stationary SMBH case, even though there is an increasing tidal disruption rate instead of a constant value. Here the calculation with model $20\left(r_{\mathrm{t}}=5 \times 10^{-5}\right)$ is not included because the particle resolution is too low to estimate disruption rate. With the same extrapolating relation described in Section 3.3.1, we estimate that the averaged tidal disruption rate for phase I should around the order of magnitude $\sim 10^{-6} \mathrm{M}_{\odot} \mathrm{yr}^{-1}$, which is about an order of magnitude lower than stationary $\mathrm{SMBH}$ in the galactic center.

For the phase II, when the orbit of the recoiling SMBH damped to relative small region, the variations of stellar density and velocity of SMBH are smaller than phase I. Thus the difference of disruption rate between apocenters and galactic center is not so intensive. Since the tidal disruption events during entire phase I are few in our simulation, the accuracy for calculating tidal disruption rate is not very good. However, there are plenty of tidal disruption events in phase II, which provides a better accuracy to calculate the tidal disruption rate in phase II. Actually, as shown in the right part of Figure 8, there are constant disruption rates as similar as the stationary case. That is because (1) the stellar densities around SMBHs in phase II and in the stationary SMBH case are similar and (2) their loss cones are both full. 


\subsection{Dependence on Particle Number}

Since $1 \mathrm{M}$ particles do not have enough high resolution to represent a real galaxy, it is important for us to find out the dependence of our results on the particle number. Only with this considered, the direct $N$-body simulation results for dynamical properties of the recoiling SMBHs and surrounding stars can be extrapolated to real conditions in galactic nuclei. To bring this forth, a series of integrations with various $N$ and same other parameters have been done, which relative to models 01,02 and 06 with $N=50 \mathrm{~K}, 250 \mathrm{~K}, 1 \mathrm{M}$ respectively. Figure 9 shows the particle number dependence of the recoiling SMBH orbital oscillation and mass increasing for model 01,02 and 06 respectively. It can be seen that the $250 \mathrm{~K}$ and $1 \mathrm{M}$ cases have similar oscillate amplitudes in phase II, whereas the $50 \mathrm{~K}$ run can not well represent the damped evolution of the recoiling SMBH. Besides, only the calculations with $250 \mathrm{~K}$ and $1 \mathrm{M}$ particles can give relatively smooth mass increasing curve, and they all achieve to the similar final mass.

Limited by the particle resolution, we can not obtain good enough mass evolution data to fit the disruption rates in phase I both for $50 \mathrm{~K}$ and $250 \mathrm{~K}$ integrations with model 01 and 02. The only thing can be done is to calculate average tidal disruption rates for phase I and II, as discussed in Section 3.3 with Figure 5. However, those problems do not exist for all of $1 \mathrm{M}$ integrations and other $250 \mathrm{~K}$ integrations. For this reason, most of our calculations adopt $1 \mathrm{M}$ particles. As shown in Figure 5, the difference of average disruption rates between $250 \mathrm{~K}$ and $1 \mathrm{M}$ is very small, which also indicates that the loss cones are nearly full.

With $1 \mathrm{M}$ particles simulation, the mass ratio of SMBH to stars should be $\sim 10^{3}$. This ratio is smaller than the real galaxy condition which should be $\sim 10^{6}-10^{9}$. The small mass ratio between SMBH and stars may lead to a higher Brownian velocity comparing with real galaxy condition (Merritt et al. 2007). However, most of our simulations are terminated before phase III, which makes the amplitude of oscillating SMBH is greater than Brownian amplitude. Detailed discussion in Gualandris \& Merritt (2008) with series of calculations

for different particle numbers shows that the particle mass can not significantly impact the dynamical evolution for both phase I and II.

The limitation of particle number can also influnece the relaxation time $T_{\mathrm{r}}$. For a homogenous isotropic distribution with equal-mass stars, the two-body relaxation time in our simulation units is (Spitzer 1987)

$$
T_{\mathrm{r}}(r) \approx \frac{0.34 \sigma^{3}}{\rho(r) m_{*} \ln \Lambda}=\frac{0.34 N \sigma^{3}}{\rho(r) \ln \Lambda},
$$


where $\ln \Lambda$ is Coulomb logarithm and we can estimate it as

$$
\ln \Lambda \approx \ln \frac{r_{\mathrm{inf}} \sigma^{2}}{2 m_{*}}=\ln \frac{N r_{\mathrm{inf}} \sigma^{2}}{2} .
$$

When $N$ is large enough, for instance $N \gtrsim 10^{6}$, the relaxation time will be roughly proportional to $N / \ln N$. It means that our $N$-body simulation with $1 \mathrm{M}$ particles always give us a shorter relaxation time comparing with real galaxy condition. This fast relaxation in $N$-body simulation may bring troubles to our results. If the core region in our simulation has relaxed before the recoiling SMBH return, our results will departure from the real galaxy condition, because the relaxation time in a real galactic core is always very long.

To solve this problem, we calculate the relaxation time of the central core region for both the simulation model and real galaxy condition. We choose $r=r_{\text {inf }}, N=10^{6}$ and $N=4 \times 10^{10}$ in model 06 to calculate the relaxation time $T_{\mathrm{r}}\left(r_{\mathrm{inf}}\right)$. Since we know little about the exactly value of velocity dispersion $\sigma$ around $r=r_{\text {inf }}$, we range the $\sigma$ from $10^{-2}$ to 1 . For the real galaxy condition, our estimations show that, the $T_{\mathrm{r}}\left(r_{\text {inf }}\right)$ is always longer than the half period of the oscillating $\mathrm{SMBH}$. That means the core region with a real galaxy condition can not relax before the SMBH return. The same result applied to our simulations with $\sigma \gtrsim 0.06$ for $N=10^{6}$. Based on Equation (9), 0.06 corresponds to $\sim 45 \mathrm{~km} \mathrm{~s}^{-1}$, which is smaller than most of the galactic center cases. Thus we can conclude that the core region can not relax before the recoiling SMBH return both for simulation and real galaxy condition.

The limitation of particle number in $N$-body simulation may also impacts the results for the stationary SMBH. In recoil case, the oscillating SMBH always corresponds to the full loss cone status both for the simulation and the real galaxy condition. For a stationary SMBH, things will be different. Both the analytical calculations and the numerical simulations for long term evolution of tidal disruption from stationary SMBH show that the loss cone in real galaxy is empty for most of the cases (Frank \& Rees 1976; Lightman \& Shapiro 1977; Cohn \& Kulsrud 1978; Baumgardt et al. 2004). However, our calculations are just focus on a relative short period, which correspond to $\sim 10^{7} \mathrm{yr}$ for our fiducial model. During this short period, the loss cone could be roughly full. This prediction has been confirmed by our simulation results.

Based on discussions above, there are some conclusions: (1) both 250K and 1M integrations are good enough to represent dynamical evolution of the recoiling SMBH, (2) in order to investigate the tidal disruption and co-evolution of the recoiling $\mathrm{SMBH}$ with surrounding stars, it is better to use integrations with $N=1 \mathrm{M}$, (3) the small difference on average disruption rates between $250 \mathrm{~K}$ and $1 \mathrm{M}$ integrations indicates that the loss cones are nearly full, otherwise the results should change obviously with various particle numbers, (4) the particle 
number we adopt in simulation is less than the real galaxy condition, which can lead to a shorter relaxation time scale. However, it can not significantly impact our results.

\subsection{Dependence on other Parameters}

Based on the Chandrasekhar's dynamical friction theory (Chandrasekhar 1943), the dynamical friction time scale is inversely proportional to the mass of the recoiling SMBH. For this reason, a heavier SMBH should has shorter evolution time scale in phase I. In order to investigate the dependence of evolution time scale on black hole mass $M_{\mathrm{BH}}$, we run a test model 07 with $M_{\mathrm{BH}}=0.002$. Comparing with model 06 , we find that model 07 gives a shorter oscillation period in phase I, which is roughly two times smaller than that in model 06. That is consistent with the prediction of dynamical friction theory. Besides, the tidal disruption rate in model 07 is roughly two times higher than that in model 06 for both of the two phases, which is roughly consistent with Equation (21). For a galaxy with lighter SMBH, which has $M_{\mathrm{BH}}<10^{-3} M$, there will be a longer dynamical evolution time in phase I and smaller tidal disruption rate comparing with our results above. Limited by the calculation capability, simulations with the lower mass SMBH will cost so much time that we can not investigate them carefully.

We note that in the simulations with very large tidal radius, for example, $r_{\mathrm{t}}=0.05$ in model 13 and 14, rapid increase on $M_{\mathrm{BH}}$ lead to the declined disruption rates. This significant growth of $M_{\mathrm{BH}}$ is artificial, which makes the declination of disruption rate is unphysical.

When considering different density slopes, the results are quite different. The higher of $\gamma$ value is, the faster of the system evolved. Our integration with model 12 shows that the recoiling SMBH will enter phase II around $t=5$, which is more faster than evolution of SMBH in model 06 with $t \sim 50$. Thus our models for $\gamma=1.0$ and 1.5 actually reach phase III at the end of integration. That can be easily understood because a larger $\gamma$ means a denser cusp in the galactic center. For the same reason, a larger $\gamma$ also leads to a higher disruption rate.

Figure 10 shows the disruption rates with different $\gamma$ values for both the stationary and the recoiling cases. The left panel is for stationary integrations and the right panel is for the recoiling SMBH with time duration $t=60-100$. Here we can not compare the same phase for different $\gamma$ models because of their different evolving times. The results in the right panel are phase II for model 06 and phase III for model 09 and 12. It can be seen that, for both panels, there are several times differences between every two adjacent $\gamma$ values.

In order to compare with the work in the literature with higher $V_{\mathrm{ej}}$, a simulation with 
similar initial parameters comparing with model 06 has been done in model 10 , where $\gamma=1$ and $V_{\mathrm{ej}}=1.1 V_{\mathrm{esc}} \simeq 1.56$. Our result shows that there are just five particles tidally disrupted at the beginning of recoil, and following with zero event recorded for the rest of time. Because of the low particle resolution, we can only give a lower limit to the tidal disruption rate. For a galaxy with mass $M=4 \times 10^{10} \mathrm{M}_{\odot}, M_{\mathrm{BH}}=4 \times 10^{7} \mathrm{M}_{\odot}$ and $r_{1 / 2}=1 \mathrm{kpc}$, the kick velocity should be $V_{\mathrm{ej}} \sim 1000 \mathrm{~km} \mathrm{~s}^{-1}$. Thus our lower limit for tidal disruption rate is $\sim 6 \times 10^{-8} \mathrm{M}_{\odot} \mathrm{yr}^{-1}$, which is lower than $\sim 10^{-6} \mathrm{M}_{\odot} \mathrm{yr}^{-1}$ in Figure 2 of Komossa \& Merritt (2008) with similar conditions. For the bound stars, we find that there are only $\sim 10^{-3} M_{\mathrm{BH}}$ stars still bound to SMBH after the recoil, which is roughly as the same order of magnitude as the result in Merritt et al. (2009). The small amount of bound stars, low stellar density outside and poor particle resolution result in the rare disruption events in computation. To solve this problem, a series of simulations with larger particle numbers are needed.

\section{DISCUSSIONS AND CONCLUSIONS}

Supermassive binary black holes in galactic nuclei may under certain conditions get very close and coalesce due to strong emission of gravitational waves. Because of the anisotropy of GW emission, the coalescence remnant SMBH experiences a strong recoil velocity depending on spins of the original pair of black holes. In this paper we investigate the interaction and co-evolution of the recoiling SMBHs with their galactic stellar environments, using very large direct $N$-body simulations with a simplified tidal disruption scheme. It is the first numerical investigation for the evolution of the tidal disruption by an oscillating SMBHs with unbound stars included. Moreover, we discover a polarization cloud of stars surrounding the oscillating SMBHs, which is not mentioned in the literature.

As argured by Gualandris \& Merritt (2008), the dynamical evolution of the recoiling SMBH can be divided into three phases. During the phase I, the trajectory of the recoiling SMBH can be well predicted by Chandrasekhar's dynamical friction theory (Chandrasekhar 1943). The phase II begins when the SMBH's oscillation decayed to the size of galactic core, and the motion of the SMBH damps slowly. At phase III, the orbital oscillation of SMBH slowly decay to achieve a thermal equilibrium with surrounding stars. In our simulation, we obtain similar phase I and II as Gualandris \& Merritt (2008) have got. Besides, our results show that the mass fraction of bound stars relative to $\mathrm{SMBH}$ is roughly $0.7 \%$ at the beginning. This value is consistent with Gualandris \& Merritt (2008) and Merritt et al. (2009).

In addition to following the dynamical evolution of the recoiling SMBH, we estimate

the stellar tidal disruption rates by the recoiling SMBHs using direct $N$-body simulation 
together with our simple tidal disruption scheme. Our results show that the stellar tidal disruptions do not significantly change the dynamical evolution of the recoiling SMBH. The stellar disruption rate by the recoiling SMBH in phase II is $\sim 10^{-5} \mathrm{M}_{\odot} \mathrm{yr}^{-1}$, which is about the same order of magnitude of the stellar disruption rate by the stationary SMBH at a spherical galactic center. However, in phase I, the stellar tidal disruption rate is about an order of magnitude lower.

In our simulation, a spherical stellar distribution is assumed. This simplified model is not accurate to represent a real galaxy. For most of real bulges/ellipticals, their stellar distributions are non-spherical. As argued by Vicari et al. (2007), the orbital decay time scales of the recoiling SMBHs are prolonged in triaxial galaxies comparing with spherical galaxies. That is because most of the recoiling SMBHs in triaxial galaxies can not return directly to their galactic centers, where the influences of dynamical friction are the strongest. For the same reason, a non-spherical dark matter halo can also significantly increase the decay time of a recoiling SMBH (Guedes et al. 2009). Since most of the tidal disruption events happened during the SMBHs passing through the galactic centers, the triaxiality may significantly reduce the tidal disruption rate from unbound stars. To investigate the impacts of triaxiality, a good solution is to have series of simulations with triaxial stellar distributions. However, it is very complicated to generate a stationary triaxial distribution for $N$-body simulation, and the prolonged orbital decay time needs more calculation resources. Here we just follow previous works to investigate the tidal disruptions from the recoiling SMBHs in spherical stellar distribution. Further works with triaxial stellar distribution will be included in our next paper.

Actually, the triaxiality can not change our results significantly because the influence of triaxiality is moderate in galaxy models with shallow central density profiles (Vicari et al. 2007). Thus our calculations with $\gamma=0.5$ will not significantly departure from the triaxial model. Besides, in most of our simulations, the maximal displacement of the recoiling $\mathrm{SMBH}$ relative to the density center is no more than $1 \mathrm{kpc}$. That keeps the trajectory of the SMBH inside the bulge, where the impact of dark matter halo are very weak comparing with baryonic matter. For this reason, we neglect the influence of the triaxial dark matter halo which is mentioned by Guedes et al. (2009).

Our simulation for escaping SMBH does not have enough particle resolution to estimate disruption rates. That needs much larger $N$-body particle number in the future simulations. Here our integrations are focus on relatively low recoiling velocity. For the assumption that $M=4 \times 10^{10} \mathrm{M}_{\odot}$ and $r_{1 / 2}=1 \mathrm{kpc}$, recoil velocity in our fiducial model is $\sim 600 \mathrm{~km} \mathrm{~s}^{-1}$. This velocity produces the oscillating SMBH an amplitude less than the effective radius of elliptical/bulge. Our results show that most of tidal disruption events occur when the 
recoiling SMBH passes through density center, but not exact the original center because of the orbital precession. The tidal disruption rates around each apocenter are much lower. An estimate for average tidal disruption rate at about apocenters in phase I over several periods for model 06 has been done. We calculate the average disruption rate when SMBH have large distant relative to density center. The disruption rate at large distance is several times or even an order of magnitude lower than the average value over whole phase I, which implies that the rate for apocenters is one or two order of magnitude lower than that by stationary SMBHs.

Komossa \& Merritt (2008) argued that off-nuclear X-ray tidal flares could be one of the observational signatures for an escaping SMBH. Unfortunately, our simulation of model 10 with $1 \mathrm{M}$ particles and the escapable recoil velocity does not have sufficient resolution to estimate the disruption rate. We can just give a lower limit $\sim 6 \times 10^{-8} \mathrm{M}_{\odot} \mathrm{yr}^{-1}$, which does not conflict to their work with similar configurations. Besides, our model 09 with $\gamma=1.0$ and recoil velocity $\sim 600 \mathrm{kms}^{-1}$ corresponds to a tidal disruption rate $\sim 10^{-5} \mathrm{M}_{\odot} \mathrm{yr}^{-1}$. This value is similar to the result of Model 1 with $V_{\text {ej }}=500 \mathrm{~km} \mathrm{~s}^{-1}$ in Komossa \& Merritt (2008). Based on our results, an oscillating SMBH with enough large recoil velocity may also contribute to off-nuclear X-ray flares around its apocenters with the low disruption rates.

An HCSS around the recoiling SMBH in our integrations has similar mass as predicted by Merritt et al. (2009). However, we can not give more information about the star clusters limited by particle resolution. In addition, we find a larger cloud of stars around the recoiling SMBHs, which are not permanently bound to the black hole but keep a kinematic correlation to it for more than an orbital time. It can be described as polarization cloud reacting to the gravitational disturbance by the recoiling $\mathrm{SMBH}$, in other words, the echo of dynamical friction. The size and mass of the polarization clouds are comparable to ultracompact dwarf galaxies, except significantly higher velocity dispersion. The existence of such kind of system may bring difficulties for distinguishing the HCSS. More detailed properties of the polarization cloud will be studied in our following work.

Our simulations for different particle numbers show that both $250 \mathrm{~K}$ and $1 \mathrm{M}$ integrations are sufficient in following the dynamical evolution of the recoiling SMBH, but the former do not have enough resolution for investigating the tidal disruption and co-evolution of SMBH with surrounding stars. Different initial masses of the recoiling SMBHs can change the results moderately. We find that a higher galactic density slope can significantly speed up the dynamical evolution of the system, and the tidal disruption rate is also boosted.

Support for this work was provided by the Chinese national 973 program (2007CB815405), the National Natural Science Foundation of China (11073002), the Research Fund for the 
Doctoral Program of Higher Education (RFDP), and the China Scholarship Council for financial support (2009601137). RS and PB acknowledge support by NAOC CAS through the Silk Road Project and (RS) through the Chinese Academy of Sciences Visiting Professorship for Senior International Scientists, Grant Number 2009S1 - 5. We thank for partial support by Global Networks and Mobility Program of the University of Heidelberg (ZUK 49/1 TP14.8 Spurzem). The special supercomputer at the Center of Information and Computing at National Astronomical Observatories, Chinese Academy of Sciences, funded by Ministry of Finance of People's Republic of China under the grant ZDYZ2008 - 2, has been used for some of the largest simulations. The titan cluster has been used, funded by the Volkswagen Foundation under grant No. I/84678 - 680. Some numerical computation has also be done on the SGI Altix 330 system at the Astronomy Department, Peking University. We are

grateful to X.-B. Wu, Zhaoyu Li and Heling Yan for useful discussions. Many thanks are due to the referee for valuable comments.

\section{REFERENCES}

Amaro-Seoane, P., Sesana, A., Hoffman, L., Benacquista, M., Eichhorn, C., Makino, J., \& Spurzem, R. 2010, MNRAS, 402, 2308

Baker, J. G., Centrella, J., Choi, D.-I., Koppitz, M., \& van Meter, J. 2006a, Physical Review Letters, 96, 111102

Baker, J. G., Centrella, J., Choi, D.-I., Koppitz, M., van Meter, J. R., \& Miller, M. C. 2006b, ApJ, 653, L93

Baumgardt, H., Makino, J., \& Ebisuzaki, T. 2004, ApJ, 613, 1133

Begelman, M. C., Blandford, R. D., \& Rees, M. J. 1980, Nature, 287, 307

Berczik, P., Merritt, D., \& Spurzem, R. 2005, ApJ, 633, 680

Berczik, P., Merritt, D., Spurzem, R., \& Bischof, H.-P. 2006, ApJ, 642, L21

Berentzen, I., Preto, M., Berczik, P., Merritt, D., \& Spurzem, R. 2009, ApJ, 695, 455

Boylan-Kolchin, M., Ma, C.-P., \& Quataert, E. 2004, ApJ, 613, L37

Campanelli, M., Lousto, C. O., Marronetti, P., \& Zlochower, Y. 2006, Physical Review Letters, 96, 111101

Campanelli, M., Lousto, C., Zlochower, Y., \& Merritt, D. 2007, ApJ, 659, L5 
Chandrasekhar, S. 1943, ApJ, 97, 255

Chatterjee, P., Hernquist, L., \& Loeb, A. 2003, ApJ, 592, 32

Chen, X., Liu, F. K., \& Magorrian, J. 2008, ApJ, 676, 54

Chen, X., Madau, P., Sesana, A., \& Liu, F. K. 2009, ApJ, 697, L149

Cohn, H., \& Kulsrud, R. M. 1978, ApJ, 226, 1087

Colpi, M., \& Dotti, M. 2009, arXiv:0906.4339

Dehnen, W. 1993, MNRAS, 265, 250

Evans, C. R., \& Kochanek, C. S. 1989, ApJ, 346, L13

Fiestas, J., Porth, O., Berczik, P., \& Spurzem, R. 2011, MNRAS, 1694

Fiestas, J., \& Spurzem, R. 2010, MNRAS, 405, 194

Frank, J., \& Rees, M. J. 1976, MNRAS, 176, 633

Gezari, S., et al. 2008, ApJ, 676, 944

Gezari, S., et al. 2009, ApJ, 698, 1367

González, J. A., Hannam, M., Sperhake, U., Brügmann, B., \& Husa, S. 2007b, Physical Review Letters, 98, 231101

González, J. A., Sperhake, U., Brügmann, B., Hannam, M., \& Husa, S. 2007a, Physical Review Letters, 98, 091101

Gould, A., \& Rix, H.-W. 2000, ApJ, 532, L29

Gualandris, A., \& Merritt, D. 2008, ApJ, 678, 780

Guedes, J., Madau, P., Kuhlen, M., Diemand, J., \& Zemp, M. 2009, ApJ, 702, 890

Haiman, Z., Kocsis, B., \& Menou, K. 2009, ApJ, 700, 1952

Harfst, S., Gualandris, A., Merritt, D., Spurzem, R., Portegies Zwart, S., \& Berczik, P. 2007, New Astronomy, 12, 357

Herrmann, F., Hinder, I., Shoemaker, D., \& Laguna, P. 2007, Classical and Quantum Gravity, 24, 33 
Hills, J. G. 1975, Nature, 254, 295

Just, A., Khan, F. M., Berczik, P., Ernst, A., \& Spurzem, R. 2011, MNRAS, 411, 653

Komossa, S. 2002, Reviews in Modern Astronomy, 15, 27

Komossa, S., \& Bade, N. 1999, A\&A, 343, 775

Komossa, S., \& Merritt, D. 2008, ApJ, 683, L21

Komossa, S., Zhou, H., \& Lu, H. 2008, ApJ, 678, L81

Lightman, A. P., \& Shapiro, S. L. 1977, ApJ, 211, 244

Lippai, Z., Frei, Z., \& Haiman, Z. 2008, ApJ, 676, L5

Liu, F. K. 2004, MNRAS, 347, 1357

Liu, F. K., \& Chen, X. 2007, ApJ, 671, 1272

Liu, F. K., \& Wu, X.-B. 2002, A\&A, 388, L48

Liu, F. K., Li, S., \& Chen, X. 2009, ApJ, 706, L133

Liu, F. K., Wu, X.-B., \& Cao, S. L. 2003, MNRAS, 340, 411

Liu, F. K., Xie, G. Z., \& Bai, J. M. 1995, A\&A, 295, 1

Liu, F. K., Zhao, G., \& Wu, X.-B. 2006, ApJ, 650, 749

Lodato, G., King, A. R., \& Pringle, J. E. 2009, MNRAS, 392, 332

Loeb, A. 2007, Physical Review Letters, 99, 041103

Madau, P., \& Quataert, E. 2004, ApJ, 606, L17

Merritt, D. 2006, Reports on Progress in Physics, 69, 2513

Merritt, D., \& Poon, M. Y. 2004, ApJ, 606, 788

Merritt, D., Berczik, P., \& Laun, F. 2007, AJ, 133, 553

Merritt, D., Milosavljević, M., Favata, M., Hughes, S. A., \& Holz, D. E. 2004, ApJ, 607, L9

Merritt, D., Schnittman, J. D., \& Komossa, S. 2009, ApJ, 699, 1690

Milosavljević, M., \& Phinney, E. S. 2005, ApJ, 622, L93 
O’Leary, R. M., \& Loeb, A. 2009, MNRAS, 395, 781

O'Leary, R. M., \& Loeb, A. 2011, arXiv:1102.3695

Peres, A. 1962, Physical Review, 128, 2471

Peters, P. C. 1964, Physical Review , 136, 1224

Pretorius, F. 2005, Physical Review Letters, 95, 121101

Rees, M. J. 1988, Nature, 333, 523

Schnittman, J. D., \& Krolik, J. H. 2008, ApJ, 684, 835

Sesana, A., Haardt, F., \& Madau, P. 2008, ApJ, 686, 432

Shields, G. A., \& Bonning, E. W. 2008, ApJ, 682, 758

Sillanpaa, A., Haarala, S., Valtonen, M. J., Sundelius, B., \& Byrd, G. G. 1988, ApJ, 325, 628

Spitzer, L. 1987, Princeton, NJ, Princeton University Press, 1987, 191 p.

Spurzem, R., et al. 2009, Computer Science - Research and Development, Volume 23, Numbers 3-4, 231

Stone, N., \& Loeb, A. 2011, MNRAS, 412, 75

Valtonen, M. J., et al. 2008, Nature, 452, 851

Vicari, A., Capuzzo-Dolcetta, R., \& Merritt, D. 2007, ApJ, 662, 797

Volonteri, M., Haardt, F., \& Madau, P. 2003, ApJ, 582, 559

Yu, Q. 2002, MNRAS, 331, 935 


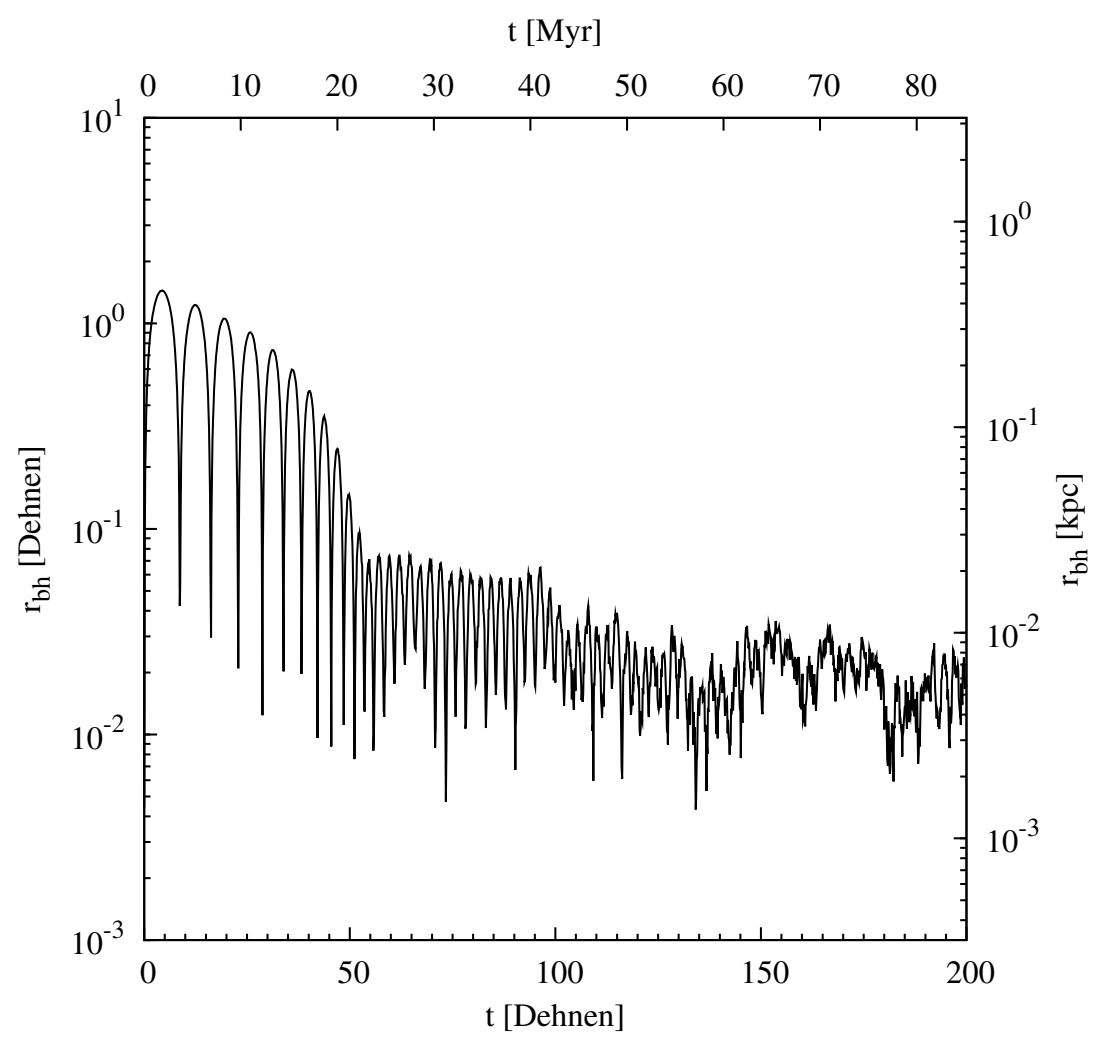

Fig. 1. - Trajectory of the recoiling SMBH with model 06. Here the bottom horizontal axis and the left vertical axis give the evolved time and the displacement of $\mathrm{BH} r_{\mathrm{bh}}$ respectively, with the units $G=M=a=1$. The right and the top axis are transformed to real physical units with the assumption that $M=4 \times 10^{10} \mathrm{M}_{\odot}$ and $r_{1 / 2}=1 \mathrm{kpc}$. It can be easily distinguished for the phase I and II which are similar to the result in Gualandris \& Merritt (2008). 


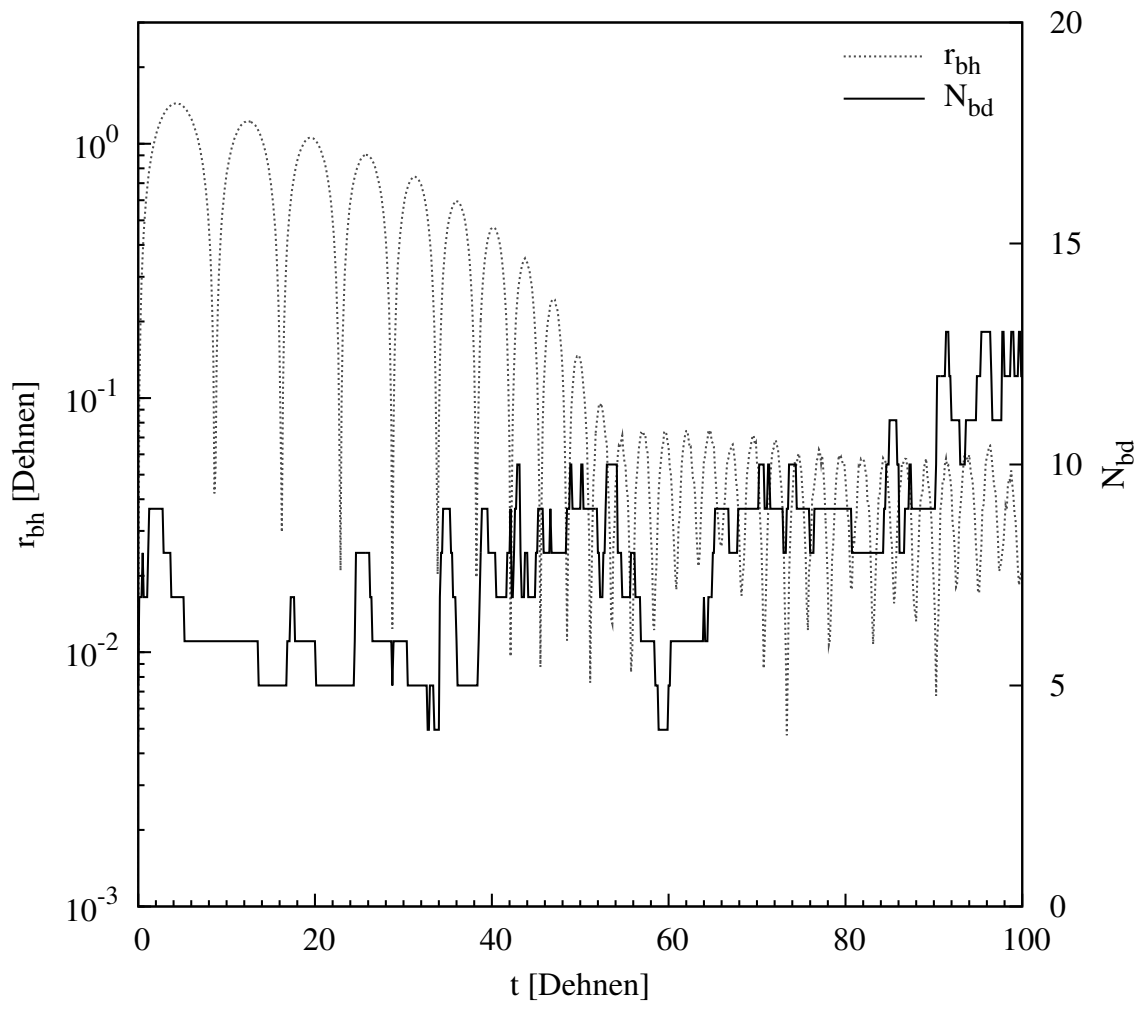

Fig. 2.- The evolution of bound star numbers around a recoiling SMBH based on the model 06. The horizontal and vertical axis are the same as Figure 3, the right horizontal axis is the number count of the bound particles. The grey dotted line gives the trajectory of the recoiling $\mathrm{SMBH}$, and the black solid line gives the variation of the bound particles. 


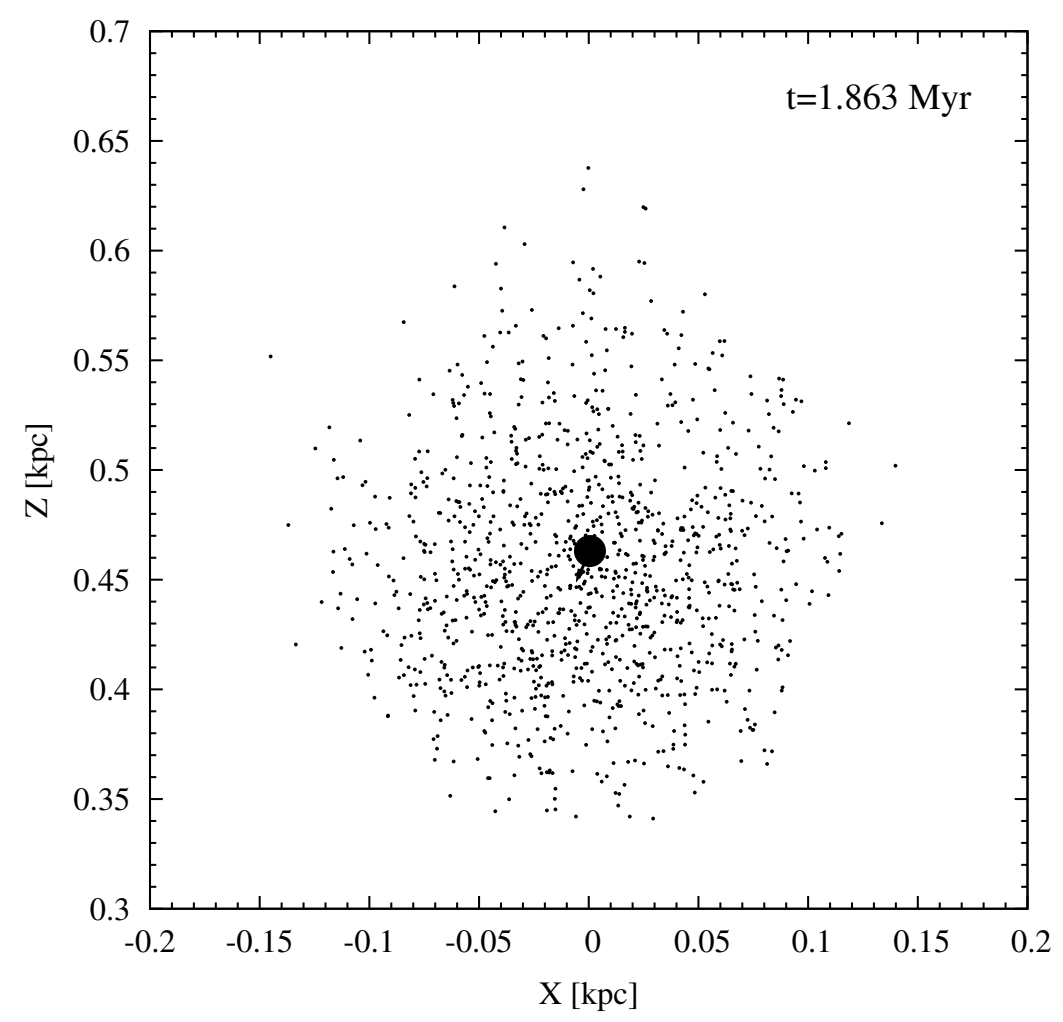

Fig. 3.- Distribution of stars which are just strongly impacted by the recoiling SMBH at its first apocenter with model 06 for time snapshot $t=1.863 \mathrm{Myr}$. We project the stellar distribution onto $X-Z$ plane, with the physical units under the assumption that $M=4 \times 10^{10} \mathrm{M}_{\odot}$ and $r_{1 / 2}=1 \mathrm{kpc}$. The large black spot marks the position of the recoiling $\mathrm{SMBH}$. It can be seen that all of the impacted star particles form a cloud structure around the SMBH at that moment. 

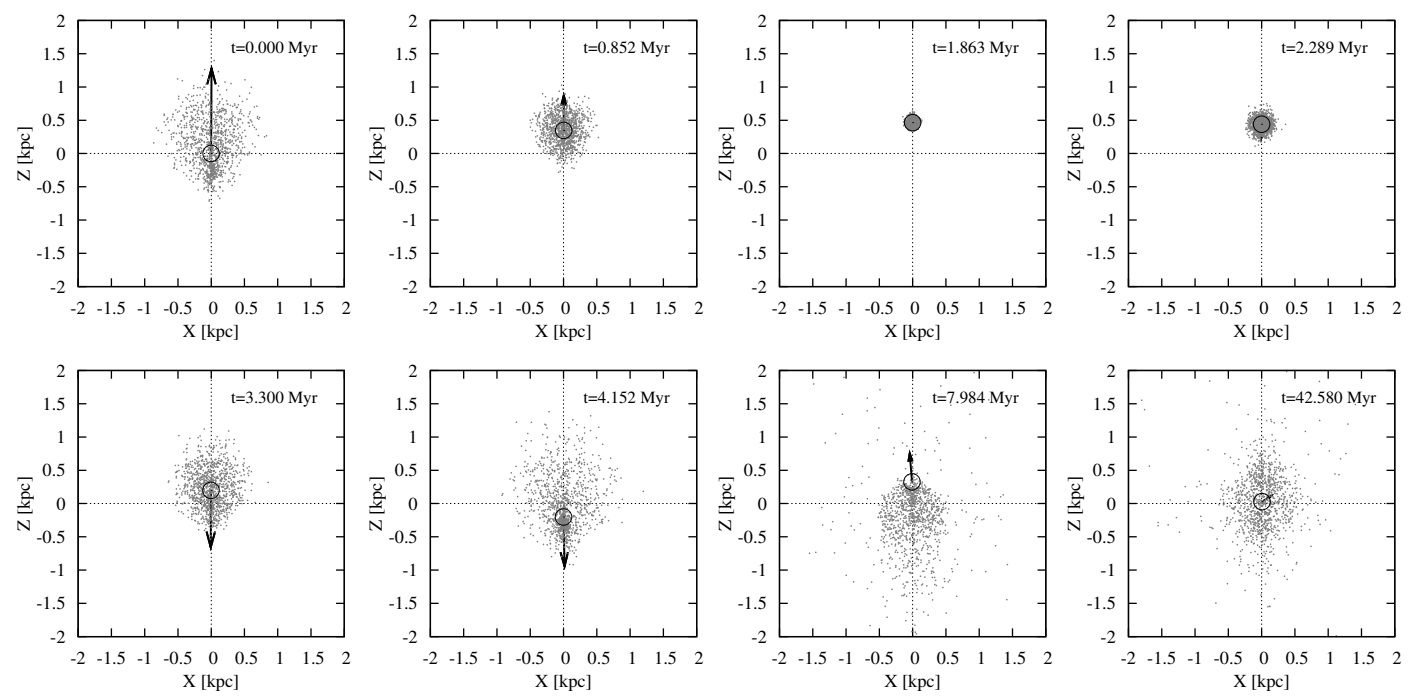

Fig. 4. - Evolution of stars which are just strongly impacted by the recoiling SMBH at its first apocenter with model 06. The units and definition of axis are the same as Figure 3 . The black circle and the grey dots mark the recoiling SMBH and the stars respectively. The black arrow represents the size and orientation of BH's velocity. It can be seen that many unbound stars are influenced by the SMBH. 

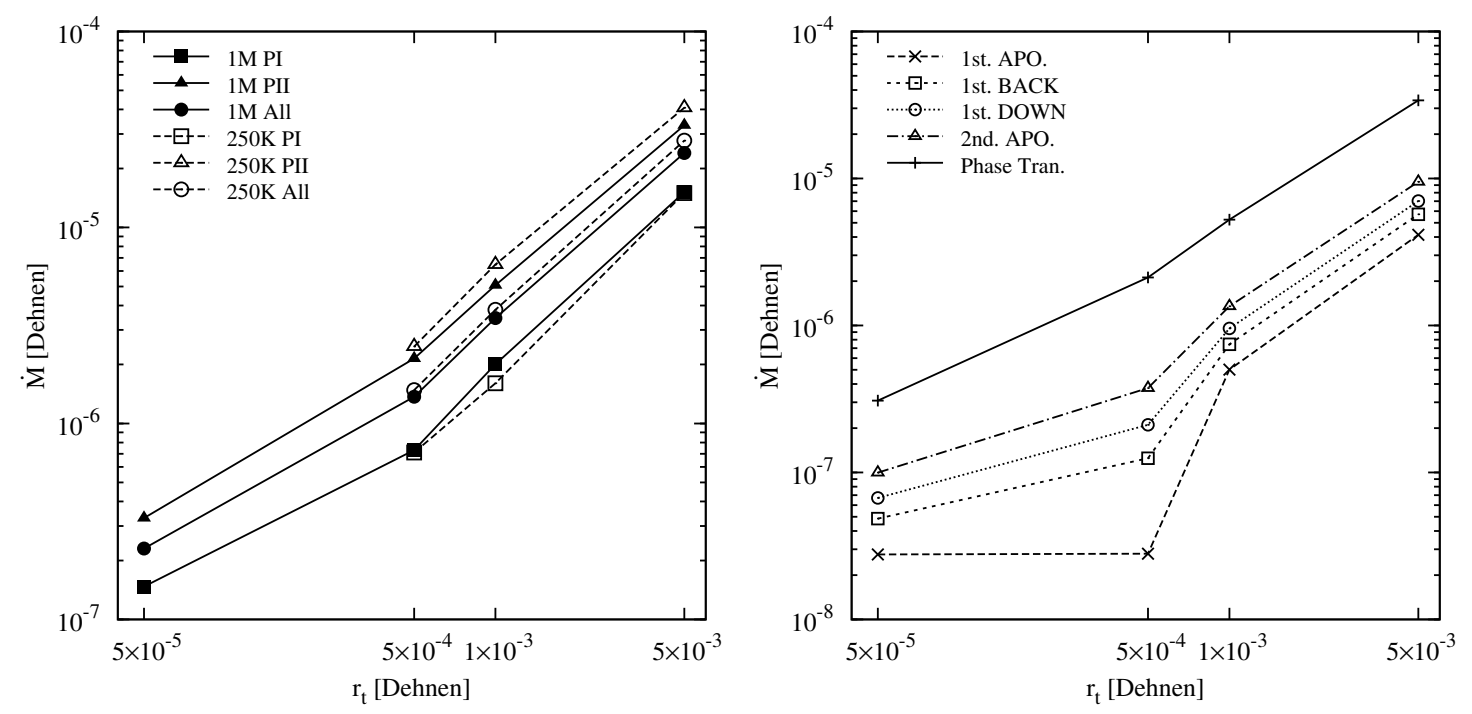

Fig. 5.- Dependence of the tidal disruption rate on $N$ and $r_{\mathrm{t}}$. The left panel shows the dependence of the average tidal disruption rates on $N$ and $r_{\mathrm{t}}$. The solid line and dashed line are the average disruption rates for $1 \mathrm{M}$ and $250 \mathrm{~K}$ integrations respectively. The square, triangle, and circles respect to the average disruption rates for phase I, phase II and the entire process respectively. The integrations are based on model 06 with different particle numbers and tidal radius. The integration for $r_{\mathrm{t}}=5 \times 10^{-5}$ with $N=250 \mathrm{~K}$ is not included because the amount of resulted tidal disruption events is too small to calculate an average rate. The right panel shows the disruption rate $-r_{\mathrm{t}}$ dependence for several key points with integrations for $N=1 \mathrm{M}$. Here "1st. APO.", "1st. BACK", "1st. DOWN", "2nd APO." and "Phase Tran." are corresponding to the moment which SMBH the first time arrive at its apocenter, the first time come back to the density center, the first time arrive at apocenter on the other side, the second time arrive at its apocenter and the transition from phase I to phase II respectively. For both of two panels, horizontal and vertical axis give the tidal radius and tidal disruption rate in the units of $G=M=a=1$ respectively. 


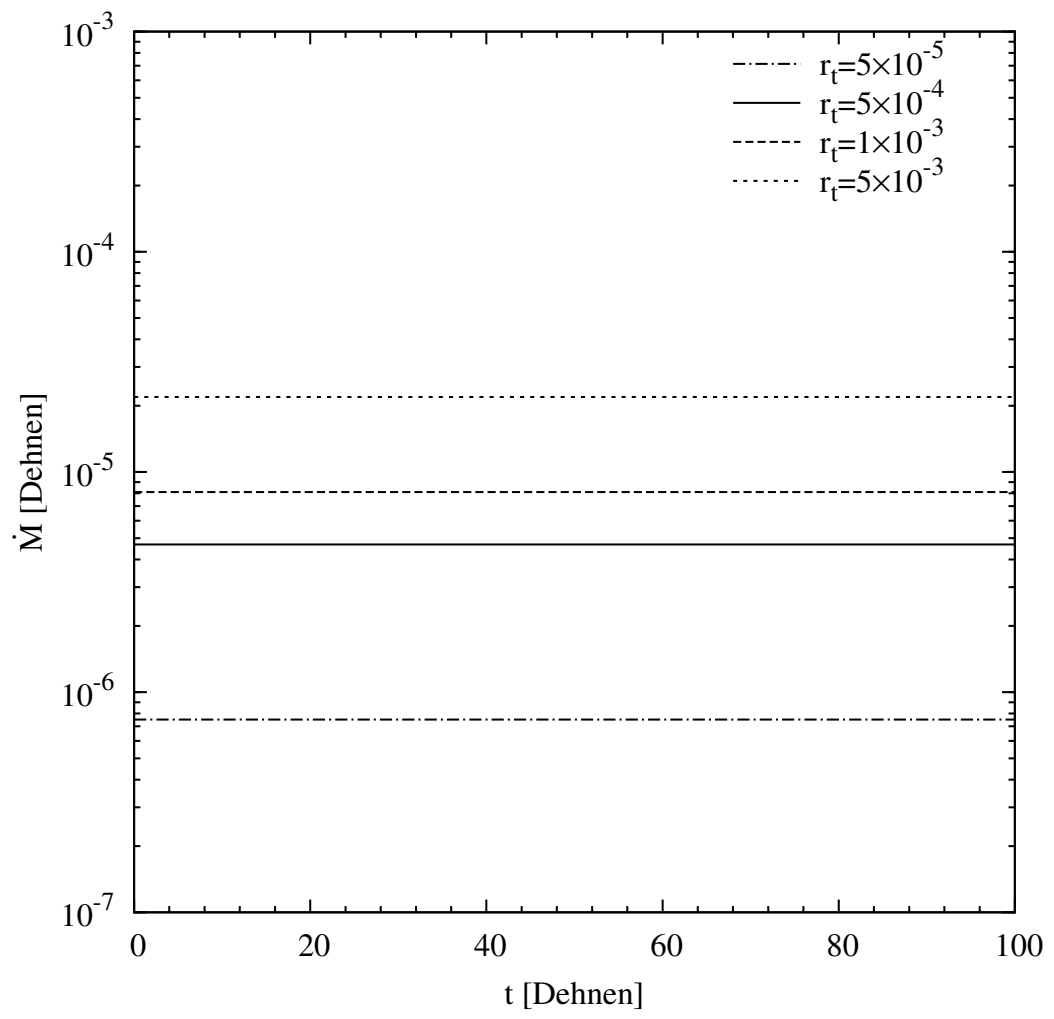

Fig. 6. - Tidal disruption rates for the stationary SMBH with different $r_{\mathrm{t}}$. Here the horizontal and vertical axis give the evolved time and tidal disruption rate in the units of $G=M=a=1$ respectively. The dotted, dashed, solid and dash dotted lines are calculations with different tidal radii $r_{\mathrm{t}}=5 \times 10^{-3}, 1 \times 10^{-3}, 5 \times 10^{-4}, 5 \times 10^{-5}$, which correspond to model 15,17, 05 and 19 respectively. 


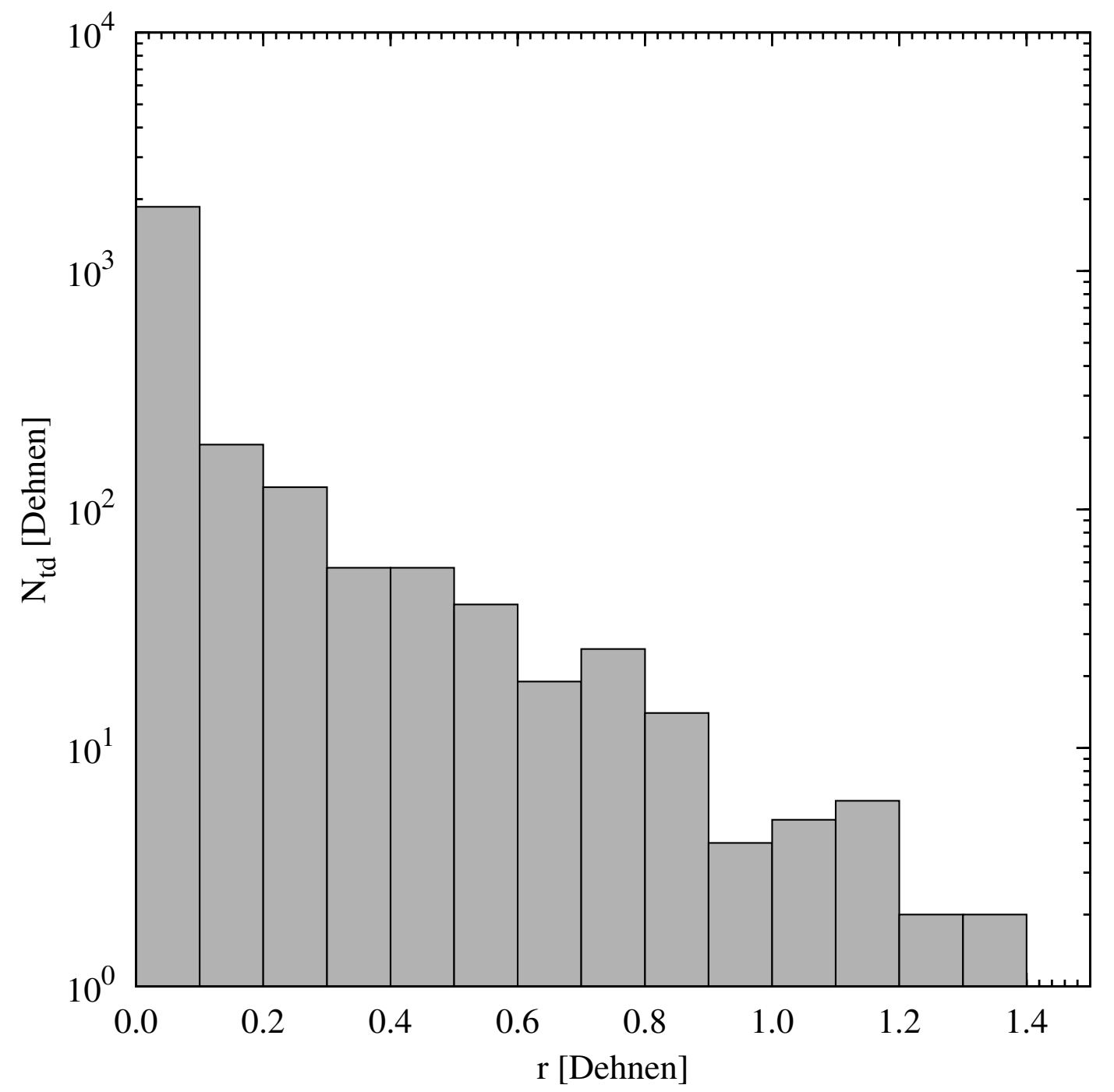

Fig. 7.- The distribution of the cumulative tidal disruption events count $N_{\text {td }}$ relative to $r$ with model 16. Here the horizontal and vertical axis give the cumulative tidal disruption events count and $r$ respectively. The shaded histogram marks the cumulative tidal disruption events count for the different distance. 


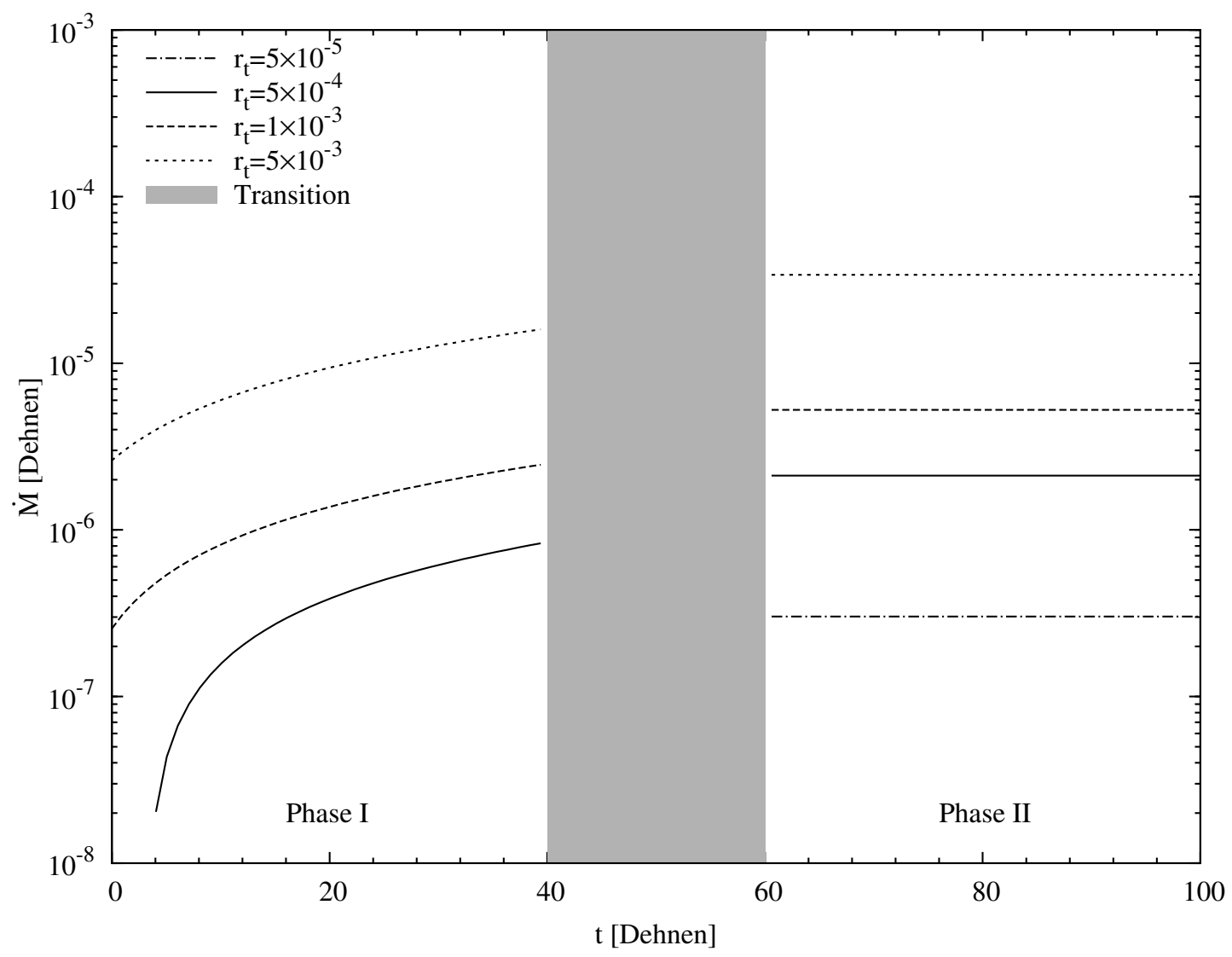

Fig. 8. - The time evolution of the tidal disruption rates with different $r_{\mathrm{t}}$. The left part and right part are the fitted results for phase I and phase II respectively. The shaded region corresponds to a transition area from phase I to phase II. Axis are labeled as same as Figure 6. The dotted, dashed, solid and dash dotted lines are the calculations with different tidal radii $r_{\mathrm{t}}=5 \times 10^{-3}, 1 \times 10^{-3}, 5 \times 10^{-4}, 5 \times 10^{-5}$, corresponding to model 16, 18, 06 and 20 respectively. Since the model 20 with $r_{\mathrm{t}}=5 \times 10^{-5}$ does not have enough particle resolution to calculate the disruption rate during phase I, it is absent in the left part. 

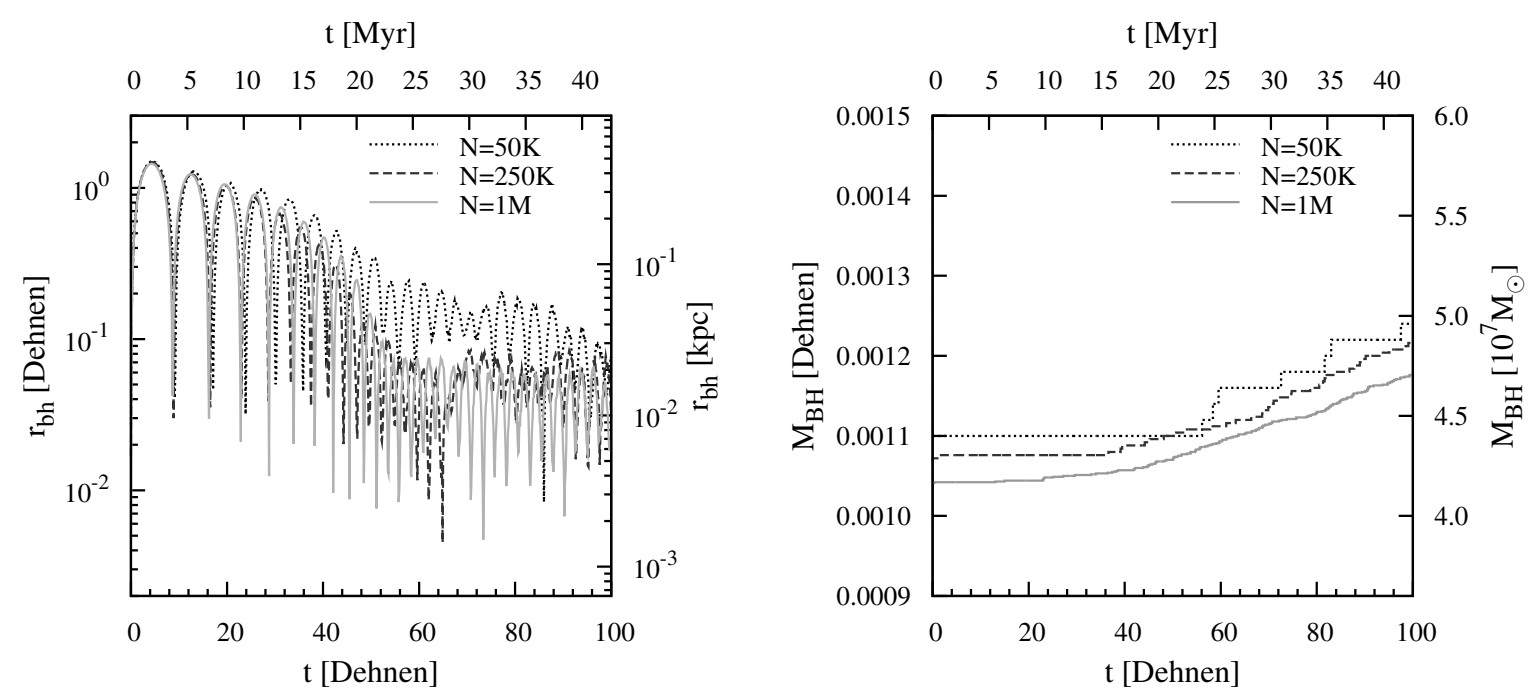

Fig. 9.- The particle number dependence for the recoiling SMBH orbital oscillation and the mass increasing with model 01, 02 and 06. Axis are labeled as same as in Figure 1. The dotted, dashed and solid lines are the calculations with particle numbers $N=50 \mathrm{~K}, 250 \mathrm{~K}, 1 \mathrm{M}$ respectively. Left panel is the damped orbital evolutions of recoiling SMBHs with various $N$. It can be seen that the $250 \mathrm{~K}$ and $1 \mathrm{M}$ cases have a similar oscillate amplitude in phase II, while the $50 \mathrm{~K}$ is different from others because of the poor particle resolution. The right panel shows the mass increasing for the recoiling SMBH. Here the vertical axis represents to the mass of the recoiling SMBH. The different initial mass of the recoiling SMBH is due to the special relaxation scheme described in Section 2.3. Only the calculations with $250 \mathrm{~K}$ and $1 \mathrm{M}$ particles can give us relatively smooth mass increasing curve, and all the calculations achieve to similar order of magnitude for final mass. 

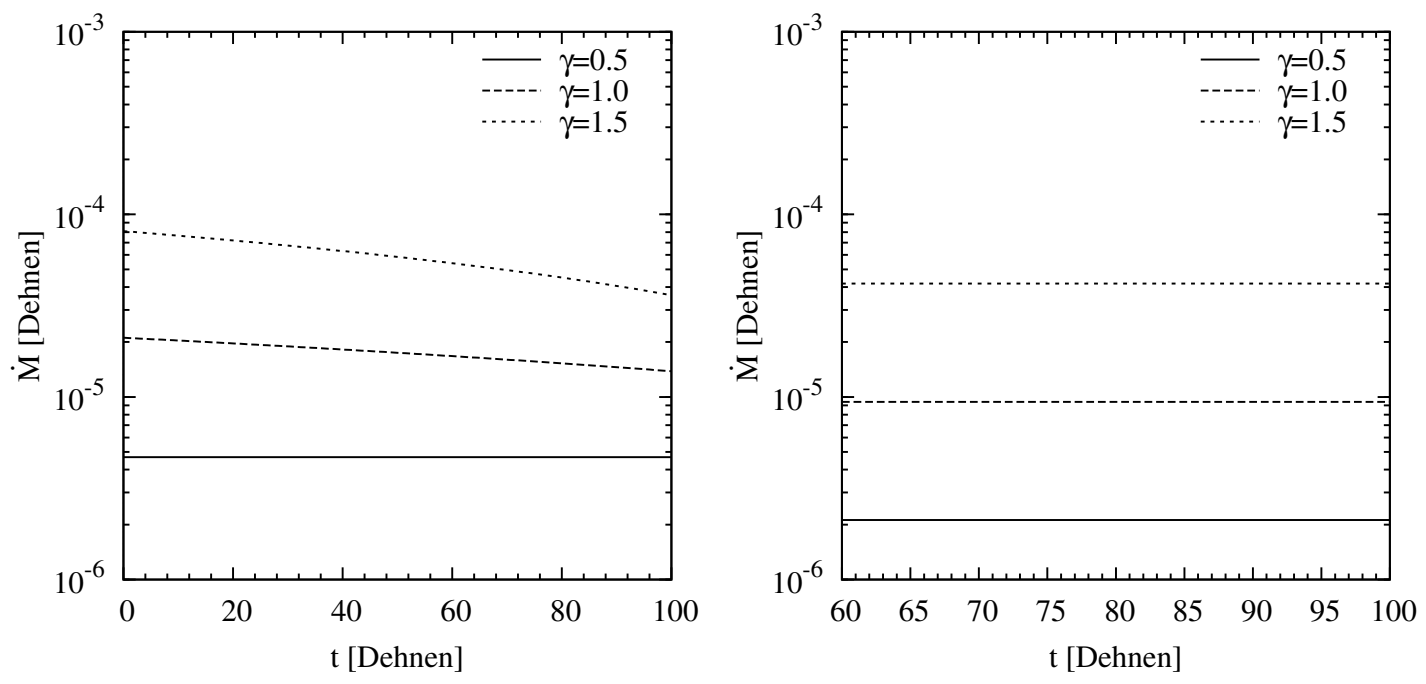

Fig. 10. - The tidal disruption rates for different $\gamma$ values and different models. The left panel is the disruption rates of stationary SMBHs with model 05, 08 and 11. The right panel shows disruption rates of the recoiling SMBHs for the time interval $t=60-100$, in model 06, 09 and 12. Here the horizontal and vertical axis stand for the evolving time and the disruption rates respectively, with the units of $G=M=a=1$. The solid, dashed and dotted lines stand for the integrations with $\gamma=0.5,1.0,1.5$ respectively. 
Table 1. Parameters of simulation models

\begin{tabular}{cccccc}
\hline \hline $\begin{array}{c}\text { Model No. } \\
(1)\end{array}$ & $N$ & $r_{\mathrm{t}}$ & $\gamma$ & $M_{\mathrm{BH} / M}$ & $V_{\mathrm{ej}} / V_{\mathrm{esc}}$ \\
$(2)$ & $(3)$ & $(4)$ & $(5)$ & $(6)$ \\
\hline 01 & $50 \mathrm{~K}$ & $5 \times 10^{-4}$ & 0.5 & 0.001 & 0.7 \\
02 & $250 \mathrm{~K}$ & $5 \times 10^{-4}$ & 0.5 & 0.001 & 0.7 \\
03 & $250 \mathrm{~K}$ & $1 \times 10^{-3}$ & 0.5 & 0.001 & 0.7 \\
04 & $250 \mathrm{~K}$ & $5 \times 10^{-3}$ & 0.5 & 0.001 & 0.7 \\
05 & $1 \mathrm{M}$ & $5 \times 10^{-4}$ & 0.5 & 0.001 & 0.0 \\
06 & $1 \mathrm{M}$ & $5 \times 10^{-4}$ & 0.5 & 0.001 & 0.7 \\
07 & $1 \mathrm{M}$ & $5 \times 10^{-4}$ & 0.5 & 0.002 & 0.7 \\
08 & $1 \mathrm{M}$ & $5 \times 10^{-4}$ & 1.0 & 0.001 & 0.0 \\
09 & $1 \mathrm{M}$ & $5 \times 10^{-4}$ & 1.0 & 0.001 & 0.7 \\
10 & $1 \mathrm{M}$ & $5 \times 10^{-4}$ & 1.0 & 0.001 & 1.1 \\
11 & $1 \mathrm{M}$ & $5 \times 10^{-4}$ & 1.5 & 0.001 & 0.0 \\
12 & $1 \mathrm{M}$ & $5 \times 10^{-4}$ & 1.5 & 0.001 & 0.7 \\
13 & $1 \mathrm{M}$ & $5 \times 10^{-2}$ & 0.5 & 0.001 & 0.0 \\
14 & $1 \mathrm{M}$ & $5 \times 10^{-2}$ & 0.5 & 0.001 & 0.7 \\
15 & $1 \mathrm{M}$ & $5 \times 10^{-3}$ & 0.5 & 0.001 & 0.0 \\
16 & $1 \mathrm{M}$ & $5 \times 10^{-3}$ & 0.5 & 0.001 & 0.7 \\
17 & $1 \mathrm{M}$ & $1 \times 10^{-3}$ & 0.5 & 0.001 & 0.0 \\
18 & $1 \mathrm{M}$ & $1 \times 10^{-3}$ & 0.5 & 0.001 & 0.7 \\
19 & $1 \mathrm{M}$ & $5 \times 10^{-5}$ & 0.5 & 0.001 & 0.0 \\
20 & $1 \mathrm{M}$ & $5 \times 10^{-5}$ & 0.5 & 0.001 & 0.7 \\
\hline
\end{tabular}

Note. - Col.(1): Model sequence number. Col.(2): Particle numbers adopted in calculations. Col.(3): Tidal disruption radius $r_{\mathrm{t}}$ in the units $G=M=a=1$. Col.(4): Density slope $\gamma$. Col.(5): Initial $\mathrm{BH}$ mass in the unit of total mass. Col.(6): Initial recoil velocity in the unit of escape velocity. All the simulations set $\epsilon=$ $10^{-5}$ and $\eta=0.01$. 